\title{
HDL-scavenger receptor B type 1 facilitates SARS-CoV-2 entry
}

\author{
Congwen Wei ${ }^{1,10}$, Luming Wan ${ }^{1,10}$, Qiulin Yan ${ }^{1,2,10}$, Xiaolin Wang ${ }^{1,10}$, Jun Zhang ${ }^{1,10}$, Xiaopan Yang ${ }^{1,10}$, \\ Yanhong Zhang', Chen Fan³, Dongyu Li', Yongqiang Deng4, Jin Sun', Jing Gong,,2, Xiaoli Yang5, \\ Yufei Wang ${ }^{5}$, Xuejun Wang6, Jianmin Li', Huan Yang ${ }^{1}$, Huilong Li', Zhe Zhang', Rong Wang', Peng Du', \\ Yulong Zong ${ }^{7}$, Feng Yin7, Wanchuan Zhang ${ }^{8}$, Nan Wang ${ }^{8}$, Yumeng Peng', Haotian Lin', Jiangyue Feng', \\ Chengfeng Qin ${ }^{4,11}$, Wei Chen ${ }^{1,11}$, Qi Gao ${ }^{9,11}$, Rui Zhang ${ }^{8,11} \bowtie$, Yuan Cao ${ }^{3,11 \bowtie}$ and Hui Zhong ${ }^{1,11} \bowtie$
}

Responsible for the ongoing coronavirus disease 19 (COVID-19) pandemic, severe acute respiratory syndrome coronavirus 2 (SARS-CoV-2) infects host cells through binding of the viral spike protein (SARS-2-S) to the cell-surface receptor angiotensin-converting enzyme 2 (ACE2). Here we show that the high-density lipoprotein (HDL) scavenger receptor B type 1 (SR-B1) facilitates ACE2-dependent entry of SARS-CoV-2. We find that the S1 subunit of SARS-2-S binds to cholesterol and possibly to HDL components to enhance viral uptake in vitro. SR-B1 expression facilitates SARS-CoV-2 entry into ACE2-expressing cells by augmenting virus attachment. Blockade of the cholesterol-binding site on SARS-2-S1 with a monoclonal antibody, or treatment of cultured cells with pharmacological SR-B1 antagonists, inhibits HDL-enhanced SARS-CoV-2 infection. We further show that SR-B1 is coexpressed with ACE2 in human pulmonary tissue and in several extrapulmonary tissues. Our findings reveal that SR-B1 acts as a host factor that promotes SARS-CoV-2 entry and may help explain viral tropism, identify a possible molecular connection between COVID-19 and lipoprotein metabolism, and highlight SR-B1 as a potential therapeutic target to interfere with SARS-CoV-2 infection.

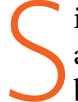
ince the outbreak of COVID-19 caused by SARS-CoV-2, approximately 1 million people have died and the total number of global confirmed cases was over 30 million as of 28 September $2020^{1}$. Coronavirus tropism is predominantly determined by the interaction between coronavirus spike $(S)$ proteins and their corresponding host receptors ${ }^{2,3}$. The S protein of SARS-CoV-2 (SARS-2-S) can be cleaved into the S1 (SARS-2-S1) and S2 (SARS2-S2) subunits, which are responsible for receptor recognition and membrane fusion ${ }^{4}$. Binding of SARS-CoV-2 to ACE2 occurs via the C-terminal domain (also called the receptor-binding domain (RBD)) of SARS-2-S1 (ref. ${ }^{5}$ ). The observed incomplete inhibition mediated by ACE2 antibodies and the high neutralization potency of monoclonal antibodies (mAbs) targeting the $\mathrm{N}$-terminal domain (NTD) of SARS-2-S1 suggest that SARS-CoV-2 might use other (co)receptors or other mechanisms for host cell entry ${ }^{6}$.

SR-B1 is a cell-surface HDL receptor that mediates the selective uptake of cholesteryl esters and other lipid components of receptor-bound HDL particles 7 . This cholesterol delivery system is well recognized in isolated hepatocytes, fibroblasts, adipocytes, macrophages, adrenal cells, ovarian cells and testicular Leydig cells ${ }^{8}$. Interestingly, alveolar type II cells also express SR-B1, where it is responsible for vitamin $\mathrm{E}$ uptake, preferentially from $\mathrm{HDL}^{9,10}$. SR-B1 has emerged as a critical receptor that affects HCV entry ${ }^{11}$; however, a potential role of SR-B1 in SARS-CoV-2 infection is unknown.
}

\section{Results}

The SARS-CoV-2 S protein binds to cholesterol. Due to the importance of SARS-2-S in mediating the infection of target cells, we conducted a search for cholesterol-regulated motifs in the primary sequence of SARS-2-S and identified six putative cholesterol recognition amino acid consensus (CRAC) motifs adjacent to the inverted cholesterol recognition motif known as CARC ${ }^{12}$ (Fig. 1a). We then evaluated the ability of SARS-2-S to associate with cholesterol. Microscale thermophoresis (MST) assay showed that SARS2-S could bind to cholesterol (Fig. 1b), but did not bind campesterol or epicholesterol, two structurally distinct sterols with additional methyl and hydroxyl groups (Fig. 1c). In a competition assay, unlabelled SARS-2-S protein had a half-maximum inhibitory concentration $\left(\mathrm{IC}_{50}\right)$ of $195.7 \pm 49.1 \mathrm{nM}$ for cholesterol (Extended Data Fig. 1a). We then evaluated the interaction of SARS-2-S, SARS2-S1 and SARS-2-S2 with HDL particles. Whereas SARS-2-S and SARS-2-S1 bound to cholesterol (Fig. 1b) and interacted either with HDL or with its components (Fig. 1d), SARS-2-S2 failed to bind to cholesterol (Fig. 1b) or HDL (Fig. 1d and Extended Data Fig. 1b). Surface plasmon resonance (SPR) assay did not provide evidence for stable binding of SARS-2-S1 to HDL, but suggested transfer of some materials from HDL to SARS-2-S1 protein (Fig. 1e). Notably, SARS-2-S did not bind to ApoA1 protein (Extended Data Fig. 1c). Together, these results support the notion

\footnotetext{
'Beijing Institute of Biotechnology, Academy of Military Medical Sciences (AMMS), Beijing, China. ${ }^{2}$ Institute of Physical Science and Information Technology, Anhui University, Hefei, China. ${ }^{3}$ Department of Basic Medical Sciences, The 960th Hospital of PLA, Jinan, China. ${ }^{4}$ State Key Laboratory of Pathogen and Biosecurity, Beijing Institute of Microbiology and Epidemiology, AMMS, Beijing, China. ${ }^{5}$ Department of Clinical Laboratory, the Third Medical Centre, Chinese PLA General Hospital, Beijing, China. ${ }^{6}$ Beijing Institute of Radiation Medicine, AMMS, Beijing, China. ${ }^{7}$ Department of Laboratory Medicine, Taian City Central Hospital Branch, Taian, China. ${ }^{8}$ Cancer Hospital of China Medical University, Liaoning Cancer Hospital and Institute, Shenyang, China. ${ }^{9}$ Beijing Hotgen Biotech Co., Ltd., Beijing, China. ${ }^{10}$ These authors contributed equally: Congwen Wei, Luming Wan, Qiulin Yan, Xiaolin Wang, Jun Zhang, Xiaopan Yang. "'These authors jointly supervised this work: Chengfeng Qin, Wei Chen, Qi Gao, Rui Zhang, Yuan Cao, Hui Zhong.

凶e-mail: zhangrui@cancerhosp-In-cmu.com; labs.net@gmail.com; towall@yahoo.com
} 
a

24-42: LPPAYTNSFTRGVYYPDKV
129-150: KVCEFQFCNDPFLGVYYHKNNK
267-277: VGYLQPRTFLL
445-461: VGGNYNYLYRLFRKSNL (RBD)
776-806: KNTQEVFAQVKQIYKTPPIKDFGGFNFSQIL
1203-1218: LGKYEQYIKWPWYIWL

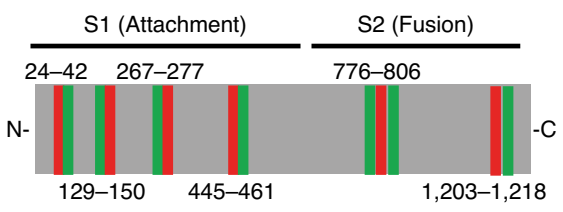

| CARC: N-R/K-X(1-5)-F/Y-X(1-5)-L/V-C

| CRAC: N-L/V-X(1-5)-Y-X(1-5)-K/R-C

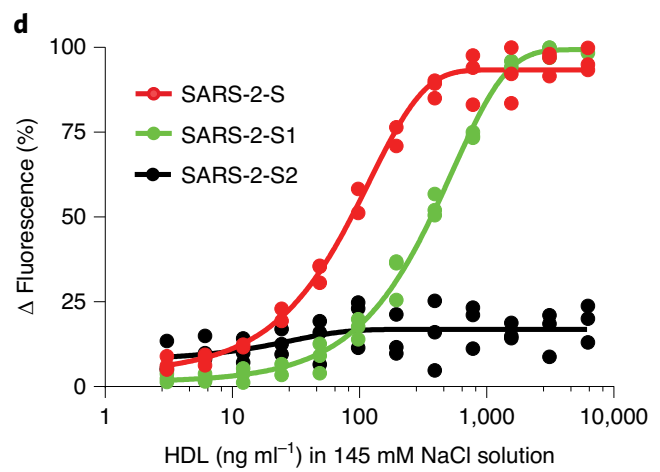

\begin{tabular}{|c|c|c|}
\hline Protein & $\mathrm{EC}_{50}\left(\mathrm{ng} \mathrm{ml}^{-1}\right)$ & $R^{2}$ \\
\hline $\mathrm{S}$ & $87.3 \pm 12.6$ & 0.98 \\
\hline $\mathrm{S} 1$ & $385.0 \pm 35.5$ & 0.99 \\
\hline
\end{tabular}

e

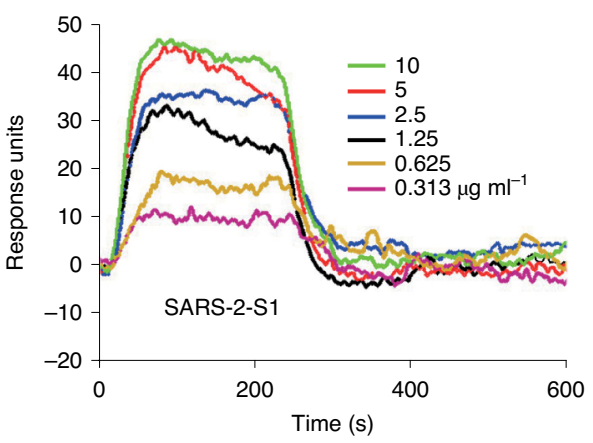

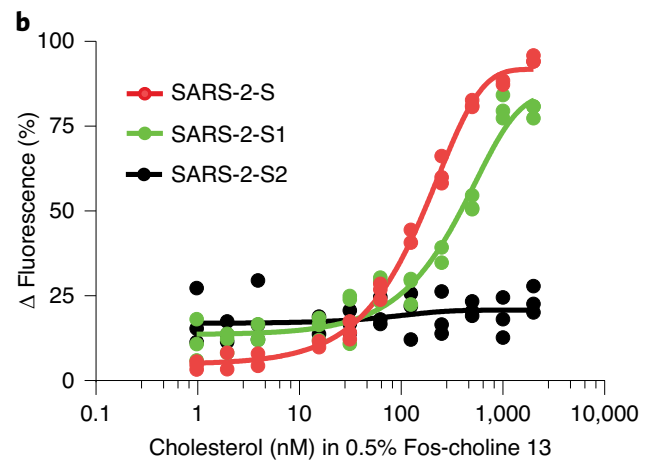

\begin{tabular}{|c|c|c|}
\hline Protein & $\mathrm{EC}_{50}(\mathrm{nM})$ & $R^{2}$ \\
\hline $\mathrm{S}$ & $154.3 \pm 37.9$ & 0.99 \\
\hline $\mathrm{S} 1$ & $489.0 \pm 51.4$ & 0.97 \\
\hline
\end{tabular}

c
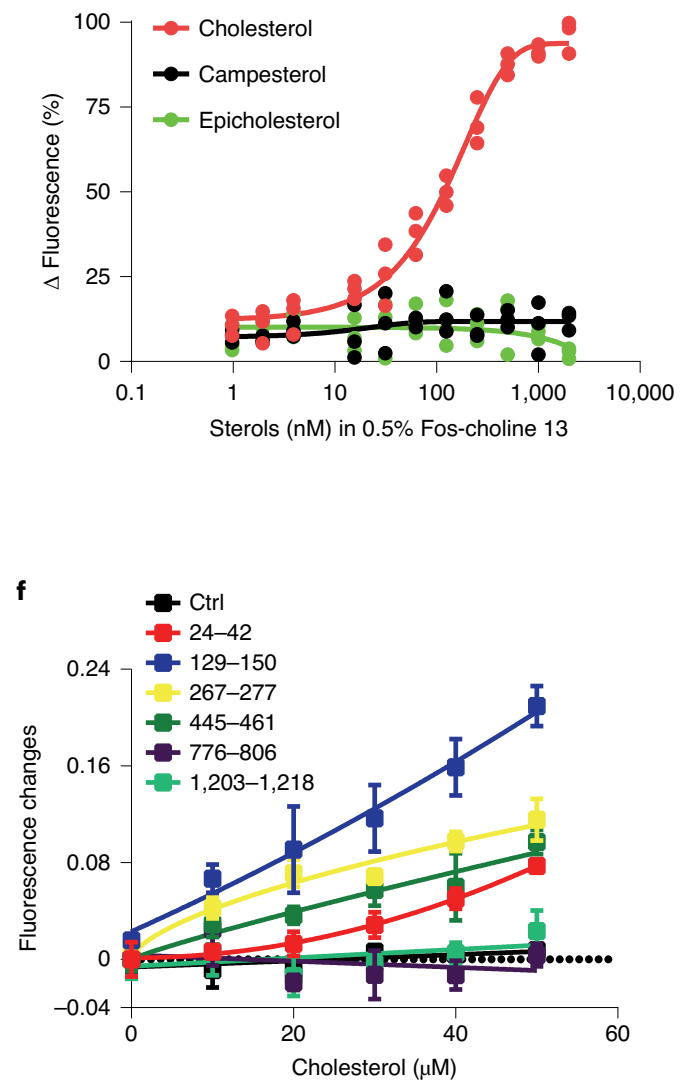

Fig. 1 | The S protein of SARS-CoV-2 binds to cholesterol. a, Schematic illustration of the cholesterol-binding motif in SARS-2-S. Crucial amino acid residues in the CARC motif are highlighted in green, crucial amino acid residues in the CRAC motif are highlighted in red and shared amino acid residues are highlighted in purple. The green stick indicates the CARC motif, and the red stick indicates the CRAC motif. $\mathbf{b}$, d, MST analysis of interactions between SARS-2-S, SARS-2-S1 or SARS-2-S2 and cholesterol (b) or HDL (d). The data were derived from the effect of cholesterol or HDL on the fluorescence decay of fluorescently labelled proteins. The half-maximum effective concentration $\left(E C_{50}\right.$ ) was determined by the Hill slope. $n=3$ independent biological experiments. c, MST analysis of interactions between SARS-2-S and cholesterol, campesterol or epicholesterol. The data were derived from the effects of the sterols on the fluorescence decay of fluorescently labelled proteins. The $\mathrm{EC}_{50}$ was determined by the Hill slope. $n=3$ independent biological experiments. e, Kinetic profile of interactions between SARS-2-S1 and HDL by SPR analysis. f, Titration curves displaying changes in the intrinsic fluorescence of the CARC-CRAC peptides $(5 \mu \mathrm{M})$ in the presence of increasing concentrations of cholesterol. $n=3$ independent biological experiments. The data are the mean \pm s.e.m.

that the SARS-2-S1 has specific affinities for cholesterol and possibly HDL components. We then attempted to dissect the interacting determinants in SARS-2-S1 through an in vitro binding assay.
Among the four cholesterol-binding peptides encompassing the CARC-CRAC region, three putative cholesterol recognition regions were observed to reside in the NTD of SARS-2-S1, while one motif 
was located in the RBD (SARS-2-RBD) (Fig. 1f). Consistent with this finding, SARS-2-RBD bound to cholesterol and HDL, or its components, in the MST assay (Extended Data Fig. 1d,e).

HDL enhances the entry of SARS-CoV-2 through SARS-2-S1 protein. We then sought to determine whether the association of SARS2-S with HDL affects SARS-CoV-2 entry. To this end, the capacity of HDL to affect the entry of SARS-2-S-pseudovirus (SARS-CoV-2pp) was explored (Extended Data Fig. 2a). HDL enhanced SARS-CoV2 pp infection in a dose-dependent manner (Fig. 2a). In addition, HDL significantly increased authentic SARS-CoV-2 viral entry (Fig. 2b) and replication (Fig. 2c). To investigate the role of HDL in SARS-CoV-2 cell-surface attachment, cells were incubated with SARS-CoV-2 at $4{ }^{\circ} \mathrm{C}$ for $10 \mathrm{~min}$ and immunolabeled for SARS-2-S after washing. HDL induced a substantial increase in cell-surface SARS-CoV-2 attachment (Fig. 2d,e). Flow cytometry also revealed that HDL significantly increased cell-surface SARS-2-S binding (Fig. 2f,g and Extended Data Fig. 2b).

In a previous study, the mAb 1D2 was isolated from patients convalescing from COVID-19 and was shown to exhibit high neutralization potency against SARS-CoV-2 (ref. ${ }^{6}$ ). Lys147, Lys150 and Tyr145, which are located in the cholesterol-binding motif of SARS-2-S, were identified as important antigenic sites for recognition by $1 \mathrm{D} 2$ ( ref. $^{6}$ ). We thus examined the ability of this $\mathrm{mAb}$ to interfere with HDL binding to SARS-2-S1. Preincubation of 1D2 with SARS-2-S1 before the addition of HDL completely blocked the SARS-2-S1-HDL binding signal in the MST assay (Fig. 2h) and strongly reduced HDL-enhanced SARS-CoV-2 infection (Fig. 2i,j). Collectively, these data indicate that HDL enhances SARS-CoV-2 infectivity either by interacting with the NTD of SARS-2-S1 or through transfer of material from HDL to the SARS-2-S1 protein.

SR-B1 expression confers susceptibility to SARS-CoV-2 infection. As SR-B1 is a cell-surface receptor that binds HDL and plays a central role in HDL endocytosis and cholesterol efflux, we wondered whether the HDL-mediated enhancement of SARS-CoV-2 infectivity occurs through SR-B1. To test this possibility, SARS-CoV-2pp entry assays were performed using two SARS-CoV-2pp-permissive cell lines, Huh-7 and Vero E6. Huh-7 cells displayed higher surface (Extended Data Fig. 3a,b) and intracellular (Extended Data Fig. 3c)
SR-B1 and ACE2 protein levels than Vero E6 cells. Notably, ectopic SR-B1 overexpression specifically enhanced Huh-7 susceptibility to SARS-CoV-2pp, but not to the unrelated VSV-Gpp (Fig. 3a and Extended Data Fig. 3d). In contrast, SR-B1 downregulation significantly reduced HDL-mediated enhancement of infection (Fig. 3b and Extended Data Fig. 3e). When using three short interfering RNAs (siRNAs) in different combinations to achieve variable potency against SR-B1 (Extended Data Fig. 3f), SARS-CoV-2pp infectivity was inversely correlated with the efficiency of silencing (Fig. 3c). We next sought to determine whether SR-B1 overexpression renders cells more susceptible to infection with authentic SARS-CoV-2. The RNA load of SARS-CoV-2 in Huh-7 cells transfected with siSR-B1 (Extended Data Fig. 3g) was approximately ten times lower than that observed in control cells (Fig. 3d), while SR-B1 overexpression (Extended Data Fig. 3h) in Vero E6 cells potently increased SARS-CoV-2 infection (Fig. 3e).

To further assess SARS-CoV-2pp susceptibility of cultured cells, MDCK, 293T and Hepa 1-6 cells with very low SARS-CoV-2pp susceptibility and nearly undetectable SR-B1 and ACE2 expression were evaluated (Extended Data Fig. 3a-c). We challenged these cells with SARS-CoV-2pp after transfecting them with complementary DNA vectors encoding human ACE2 and/or SR-B1 (Extended Data Fig. 4a-c). MDCK (Extended Data Fig. 4d) and 293T (Fig. 3f) cells became highly susceptible to SARS-CoV-2pp upon ACE2 expression, while Hepa 1-6 cells remained less permissive (Fig. 3g). Although SR-B1 introduction did not render these cells more permissive, the combined SR-B1 and ACE2 expression resulted in higher susceptibility than that observed with ACE2 or SR-B1 expression alone (Fig. 3f,g and Extended Data Fig. 4d). In particular, SARS-CoV-2pp infectivity was correlated with SR-B1 expression levels only when ACE2 was overexpressed (Fig. 3h and Extended Data Fig. 4e). Of note, the expression of SR-B1 with or without ACE2 did not modify the cell-surface expression levels of either of these entry factors at $24 \mathrm{~h}$ after transfection (Extended Data Fig. 4b,c). Given that SR-B1 only enhances viral uptake in the presence of ACE2, our results indicate that SR-B1 is an entry cofactor of SARS-CoV-2.

SR-B1 mediates SARS-CoV-2 attachment and entry. Further analysis was performed to assess the colocalization of SARS-CoV-2pp

Fig. 2 HDL enhances the entry of SARS-CoV-2 through SARS-2-S1 protein. a, Huh-7 cells were inoculated with SARS-CoV-2pp in solutions containing $\mathrm{HDL}$ at the indicated concentrations, and pseudotyped viral entry was analysed by luciferase activity at $48 \mathrm{~h}$ after infection. Signals obtained in $0.1 \%$ FBS were used for normalization. $n=3$ independent biological experiments. $P<0.0001$ for $0.1 \%$ FBS versus HDL $1 \mu \mathrm{g} \mathrm{ml}{ }^{-1}, P<0.0001$ for $0.1 \%$ FBS versus $\mathrm{HDL} 3 \mu \mathrm{g} \mathrm{ml}^{-1}, P<0.0001$ for $0.1 \% \mathrm{FBS}$ versus HDL $6 \mu \mathrm{g} \mathrm{ml} \mathrm{l}^{-1}$ by one-way ANOVA and Bonferroni's post hoc analysis; $P<0.0001$ for $0.1 \% \mathrm{FBS}$ versus $\mathrm{HDL}$ $12 \mu \mathrm{g} \mathrm{ml}^{-1}$ by one-way ANOVA and Bonferroni's post hoc analysis. b. Huh-7 cells were infected with SARS-CoV-2 in solutions containing HDL ( $\left.1 \mu \mathrm{g} \mathrm{ml}{ }^{-1}\right)$ for $1 \mathrm{~h}$; the cells were collected $3 \mathrm{~h}$ after infection for detecting the gene copy number of the virus by RT-qPCR (left graph) or intracellular SARS-CoV-2 staining by confocal microscopy (right images). The scale bar indicates $40 \mu \mathrm{m}$. The images are representative of two independent experiments. $n=3$ independent biological experiments. $P=0.0013$ for $0.1 \%$ FBS versus HDL by two-tailed Student's $t$-tests. c, Huh-7 cells were infected with SARS-CoV-2 in solutions containing $\mathrm{HDL}$ at the indicated concentrations for $1 \mathrm{~h}$, and the cells were collected to detect the gene copy number of the virus with RT-qPCR at $24 \mathrm{~h}$ after infection. $n=3$ independent biological experiments. $P<0.0001$ for $0.1 \% \mathrm{FBS}$ versus HDL $6 \mu \mathrm{g} \mathrm{ml}^{-1}, P<0.0001$ for $0.1 \%$ FBS versus HDL $12 \mu \mathrm{g} \mathrm{ml}{ }^{-1}$ by one-way ANOVA and Bonferroni's post hoc analysis. $\mathbf{d}, \mathbf{e}$, Huh-7 cells were exposed to SARS-CoV-2 $(\mathrm{MOI}=0.1)$ in solutions with or without $\mathrm{HDL}\left(6 \mu \mathrm{g} \mathrm{ml}^{-1}\right)$ at $4{ }^{\circ} \mathrm{C}$ for $10 \mathrm{~min}$. All images were obtained by confocal microscopy. The scale bar indicates $40 \mu \mathrm{m}$. The images are representative of two independent experiments (d). The bars in the graph represent the relative fluorescence intensity (RFI) from 50 cells from three biologically independent samples and five technical duplicates for each sample. $P<0.0001$ for $0.1 \%$ FBS versus HDL by two-tailed Student's $t$-tests (e). $\mathbf{f}, \mathbf{g}$, HeLa-hACE2 cells were exposed to SARS-2-S in solutions containing $\mathrm{HDL}$ at $4{ }^{\circ} \mathrm{C}$ for $30 \mathrm{~min}$. The cells were washed and detached before immunolabeling for flow cytometry (f). The

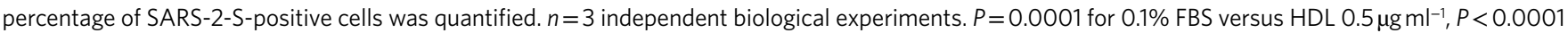
for $0.1 \% \mathrm{FBS}$ versus HDL $5 \mu \mathrm{g} \mathrm{ml} \mathrm{m}^{-1}$ by one-way ANOVA and Bonferroni's post hoc analysis ( $\left.\mathbf{g}\right)$. $\mathbf{h}, 1 \mathrm{D} 2(200 \mathrm{nM})$ was preincubated with SARS-2-S1 (100 nM),

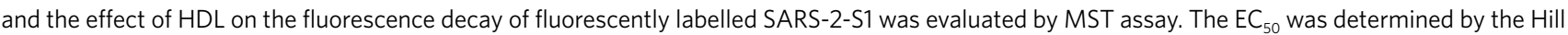
slope. $n=3$ independent biological experiments. i, Huh-7 cells preincubated with serially diluted 1D2 were inoculated with SARS-CoV-2pp in solutions containing $6 \mu \mathrm{g} \mathrm{ml}^{-1} \mathrm{HDL}$, and pseudotyped viral entry was analysed by luciferase activity at $48 \mathrm{~h}$ after infection. Signals obtained without 1D2 were used for normalization. $n=3$ independent biological experiments. $\mathbf{j}$, Huh-7 cells were infected with SARS-CoV-2 (MOI=0.001) preincubated with 1D2 (167 nM) in solutions containing $6 \mu \mathrm{g} \mathrm{m}^{-1} \mathrm{HDL}$ for $1 \mathrm{~h}$, and the cells were collected to detect the gene copy number of the virus with RT-qPCR at $24 \mathrm{~h}$ after infection. $n=3$ independent biological experiments. $P<0.0001$ for $0.1 \%$ FBS IgG versus $0.1 \%$ FBS 1D2, $P<0.0001$ for HDL IgG versus HDL $1 D 2$ by two-tailed Student's $t$-tests. This experiment was performed two times with similar results. The data are the mean \pm s.e.m. ${ }^{\star \star \star} P<0.001$. GE, genome equivalent. 
with SR-B1. Immunostaining results revealed colocalization of SR-B1 with SARS-CoV-2pp (Fig. 4a). In particular, depletion of SR-B1 significantly decreased authentic SARS-CoV-2 attachment (Fig. 4b and Extended Data Fig. 5a,b) and entry (Fig. 4c), while SARS-CoV-2 entry was greatly enhanced when SR-B1 was overexpressed (Fig. 4d and Extended Data Fig. 5a). Ectopic SR-B1 expression also led to significantly increased SARS-2-S cell-surface binding stimulated by HDL (Fig. 4e and Extended Data Fig. 5c,d). Because SARS-2-S cannot bind to SR-B1 directly (Extended Data Fig. 5e), we suggest that HDL might form a bridge between SARS-CoV-2 and SR-B1 during viral entry.

The involvement of SR-B1 was further validated by testing the effects of known pharmacological SR-B1 inhibitors, such as ITX
5061 (ref. ${ }^{13}$ ) and block lipid transport-1 (BLT-1), on SARS-CoV-2 infection. ITX 5061 is a ketoamide that inhibits HDL catabolism through targeting SR-B1 (ref. ${ }^{13}$ ). BLT-1 is a small molecule that inhibits the SR-B1-mediated selective transfer of lipids without affecting HDL-SR-B1 binding ${ }^{14}$. Both compounds exerted no unwanted cytotoxic effects (Extended Data Fig. 5f,g) and significantly blocked SARS-CoV-2pp infection (Extended Data Fig. 5h,i). Importantly, the HDL-mediated enhancement of SARS-CoV-2pp infectivity was blocked by BLT-1 and by clinical-grade ITX 5061 (Fig. 4f). To obtain direct evidence that ITX 5061 can indeed interfere with SARS-CoV-2 infection, we also infected Huh-7 cells with bona fide SARS-CoV-2 in the presence of HDL, with or without ITX
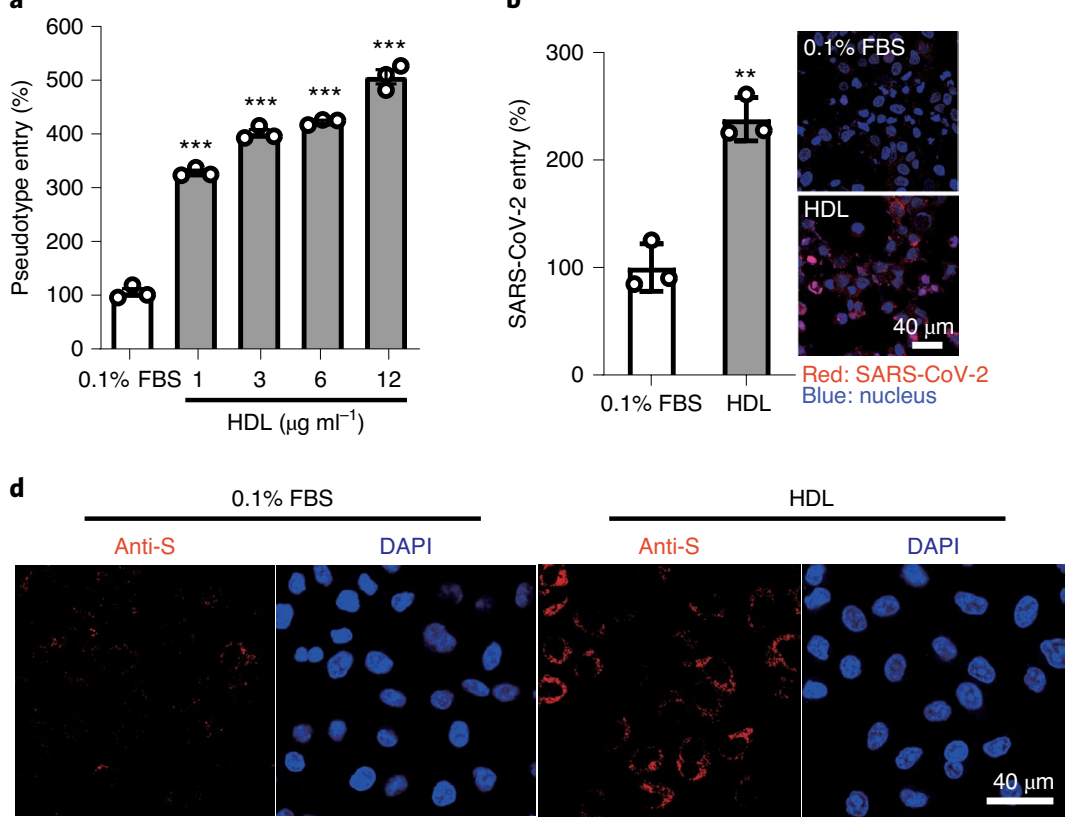

b

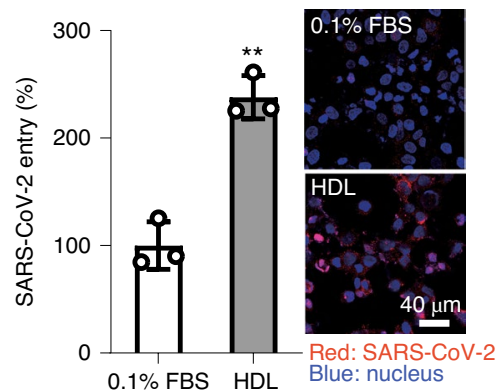

Blue: nucleus

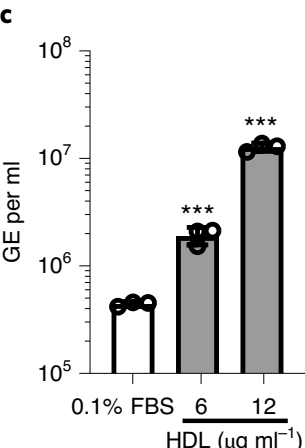

$\left.\mathrm{HDL}(\mu \mathrm{g} \mathrm{m})^{-1}\right)$
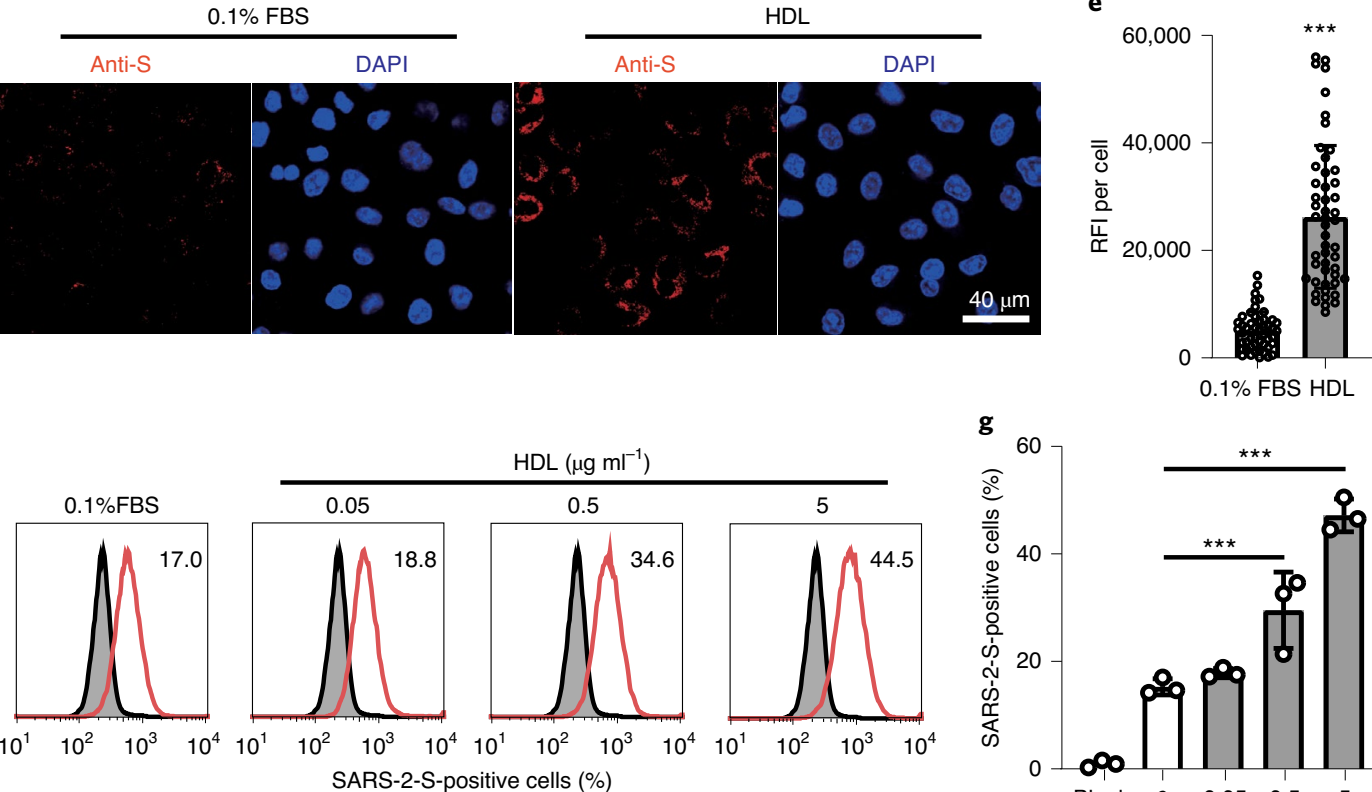

g

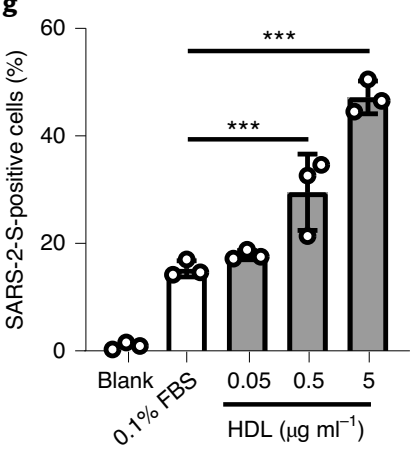

h

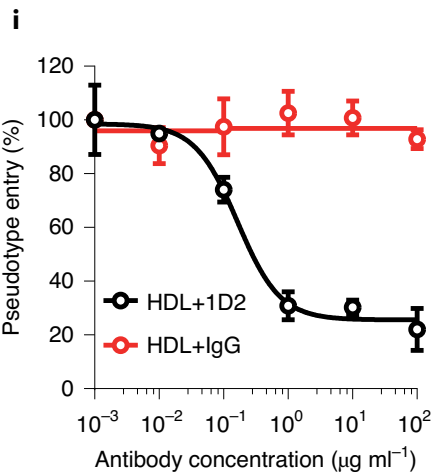

j

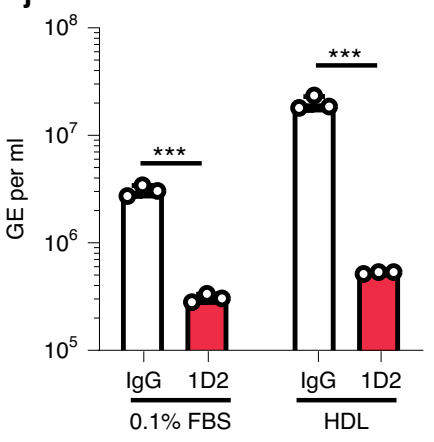




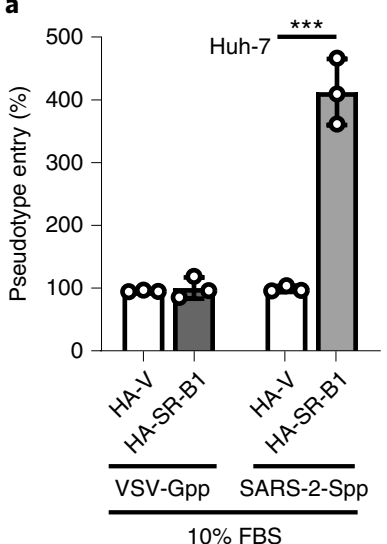

d

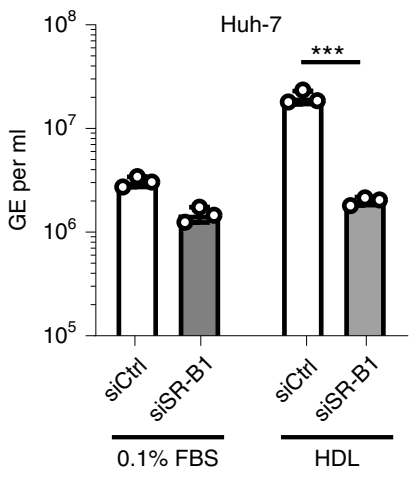

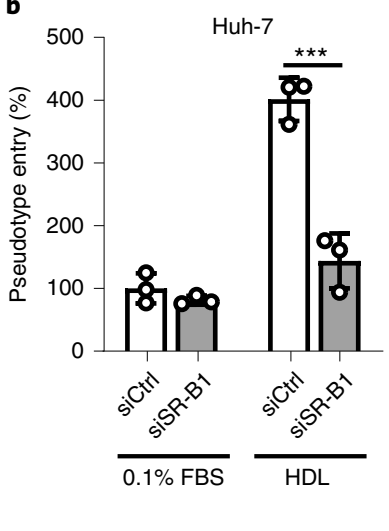

e

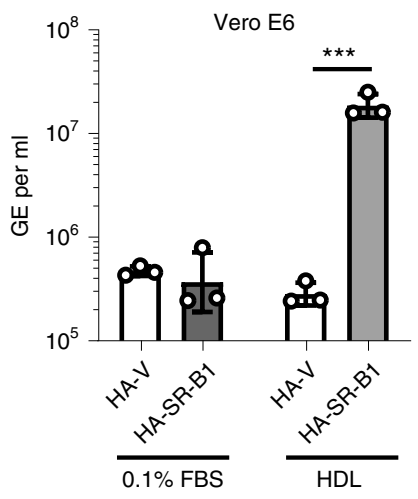

c

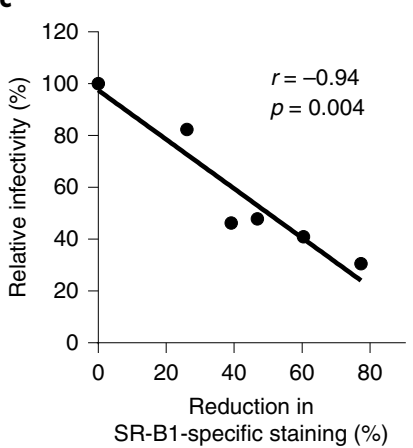

f

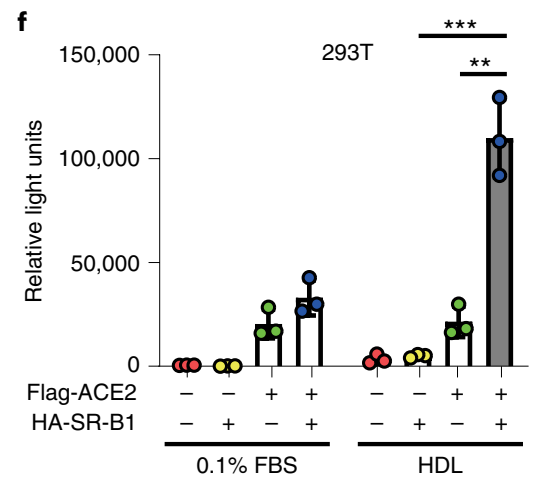

g

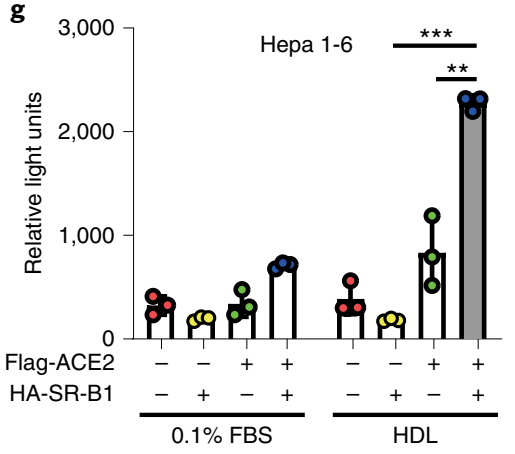

h

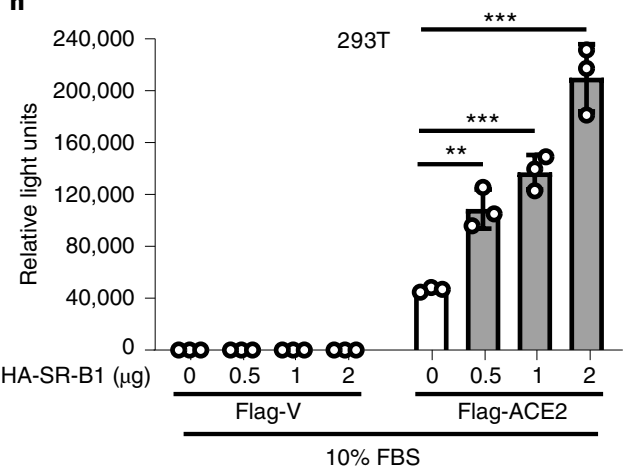

Fig. 3 I SR-B1 expression confers susceptibility to SARS-CoV-2 infection. a, Huh-7 cells transfected with HA-Vector (HA-V) or HA-SR-B1 were inoculated with SARS-CoV-2pp or VSV-Gpp in 10\% FBS, and pseudotyped viral entry was analysed by luciferase activity at $48 \mathrm{~h}$ after infection. Signals obtained in cells transfected with HA-Vector were used for normalization. $n=3$ independent biological experiments. $P<0.0001$ for SARS-2-Spp HA-V versus SARS-2-Spp HA-SR-B1 by two-tailed Student's t-tests. b, Control (siCtrl) or SR-B1-knockdown (siSR-B1) Huh-7 cells were inoculated with SARS-CoV-

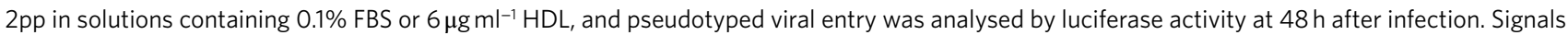
obtained in siCtrl cells with $0.1 \%$ FBS were used for normalization. $n=3$ independent biological experiments. $P<0.0001$ for $\mathrm{HDL}$ siCtrl versus $\mathrm{HDL}$ siSR-B1 by two-tailed Student's t-tests. c, Huh-7 cells were transfected with three siRNAs against SR-B1 in different combinations and then challenged with SARS-CoV-2pp in 10\% FBS. The correlation between the efficiency of SR-B1 silencing and pseudotyped viral entry normalized to that in control cells and $P=0.004, r=-0.94$ was determined by Spearman's nonparametric test. d,e, Huh-7 cells transfected with siSR-B1 (d) or Vero E6 cells transfected with a plasmid encoding SR-B1 (e) were infected with SARS-CoV-2 (MOI=0.001) in solutions containing $0.1 \%$ FBS or $6 \mu \mathrm{g} \mathrm{l}^{-1} \mathrm{HDL}$ for $1 \mathrm{~h}$. Cells were collected to detect the gene copy number of the virus with RT-qPCR after $24 \mathrm{~h} . n=3$ independent biological experiments. $P<0.0001$ for HDL siCtrl versus HDL siSR-B1, $P<0.0001$ for HDL HA-V versus HDL HA-SR-B1 by two-tailed Student's t-tests. f,g, 293 T (f) or Hepa 1-6 (g) cells were transfected with plasmids encoding ACE2, SR-B1 or both and then challenged with SARS-CoV-2pp in solutions containing $0.1 \%$ FBS or $6 \mu \mathrm{ml} \mathrm{I}^{-1} \mathrm{HDL}$. $n=3$ independent biological experiments. $P=0.0006$ for HDL SR-B1 versus HDL ACE2 + SR-B1, $P=0.0016$ for HDL ACE2 versus HDL ACE2 + SR-B1 by one-way ANOVA and Bonferroni's post hoc analysis (f). $P<0.0001$ for HDL SR-B1 versus HDL ACE2 + SR-B1, $P=0.0019$ for HDL ACE2 versus HDL ACE2 + SR-B1 by one-way ANOVA and Bonferroni's post hoc analysis $(\mathbf{g})$. h, 293T cells transfected with plasmids encoding SR-B1 together with Flag-Vector or Flag-ACE2 were inoculated with SARS-CoV-2pp in 10\% FBS, and pseudotyped viral entry was analysed by luciferase activity at $48 \mathrm{~h}$ after infection. $n=3$ independent biological experiments. $P=0.0021$ for Flag-ACE2 + HA-SR-B1 ( $0 \mu g$ ) versus Flag-ACE2 + HA-SR-B1 (0.5 $\mu$ g), $P=0.0003$ for Flag-ACE2 + HA-SR-B1 $(0 \mu \mathrm{g})$ versus Flag-ACE2 + HA-SR-B1 $(1 \mu \mathrm{g}), P=0.0004$ for Flag-ACE2 + HA-SR-B1 (0 $\mu g)$ versus Flag-ACE2 + HA-SR-B1 (2 $\mu$ g) by one-way ANOVA and Bonferroni's post hoc analysis. The data are the mean \pm s.e.m. ${ }^{\star \star} P<0.01,{ }^{\star \star \star} P<0.001 . r$, Pearson correlation coefficient. 
a

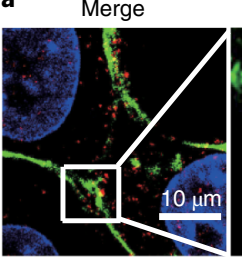

GFP-SR-B1

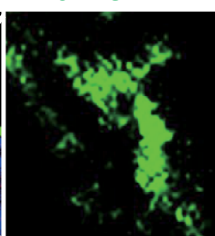

b

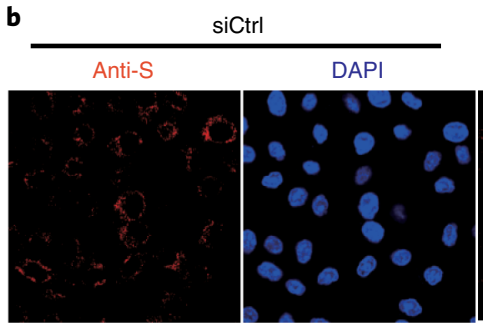

c
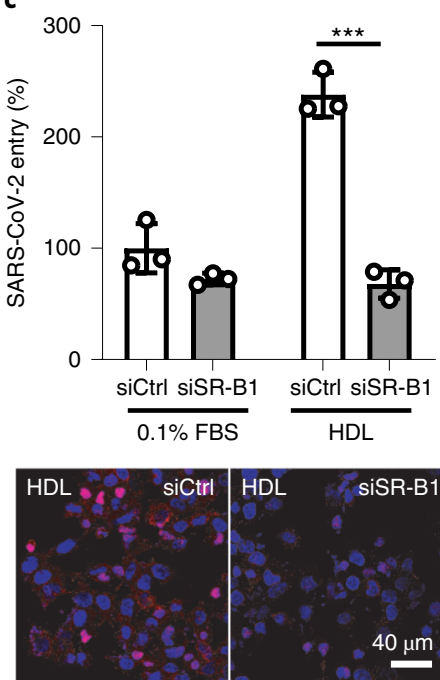

Red: SARS-CoV-2 Blue: nucleus

\section{h}

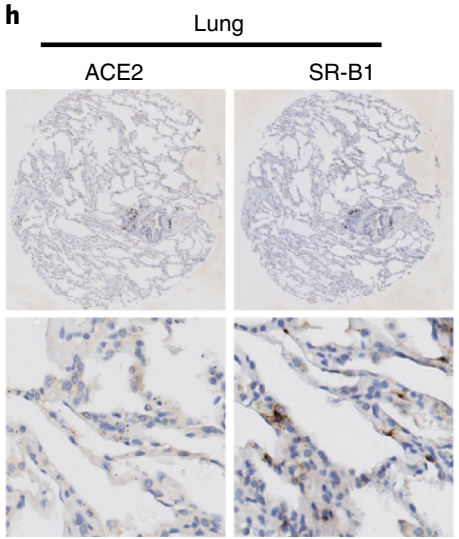

SARS-CoV-2pp
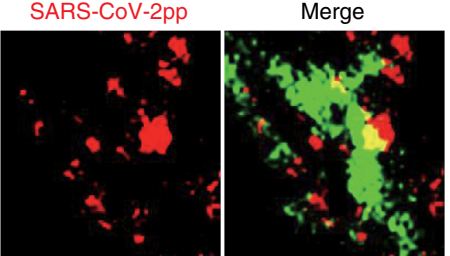

siSR-B1

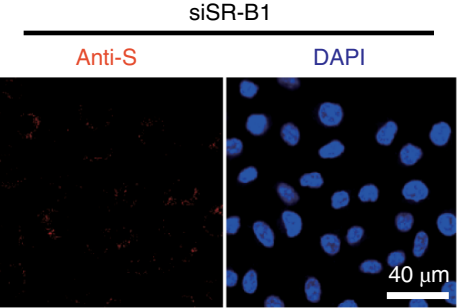

d
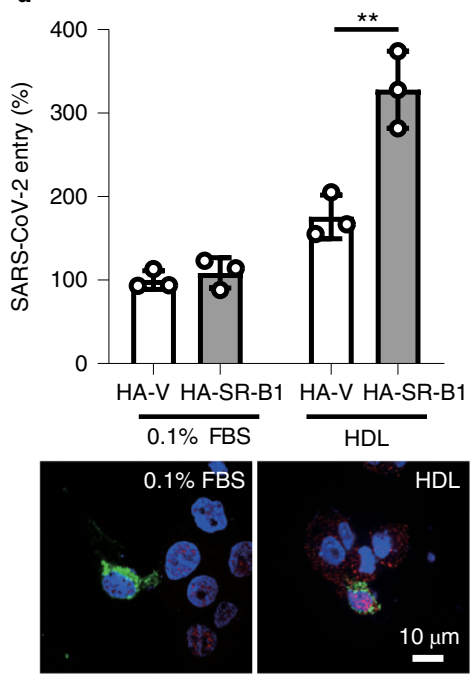

Green: SR-B1 Red: SARS-CoV-2 Blue: nucleus
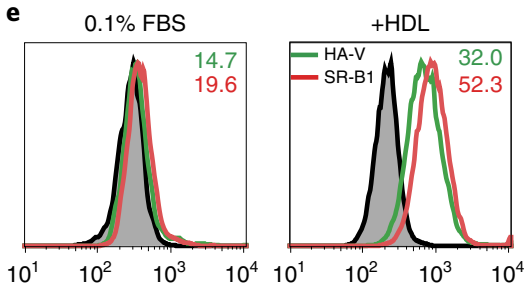

SARS-2-S-positive cells (\%)

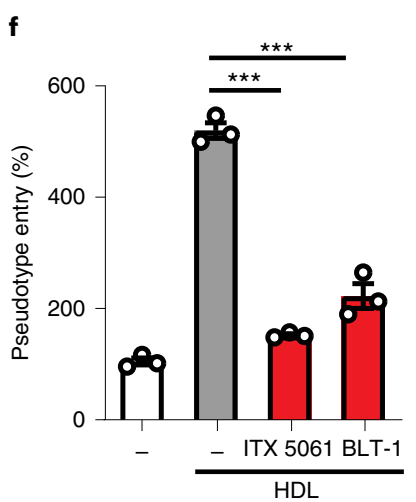

g
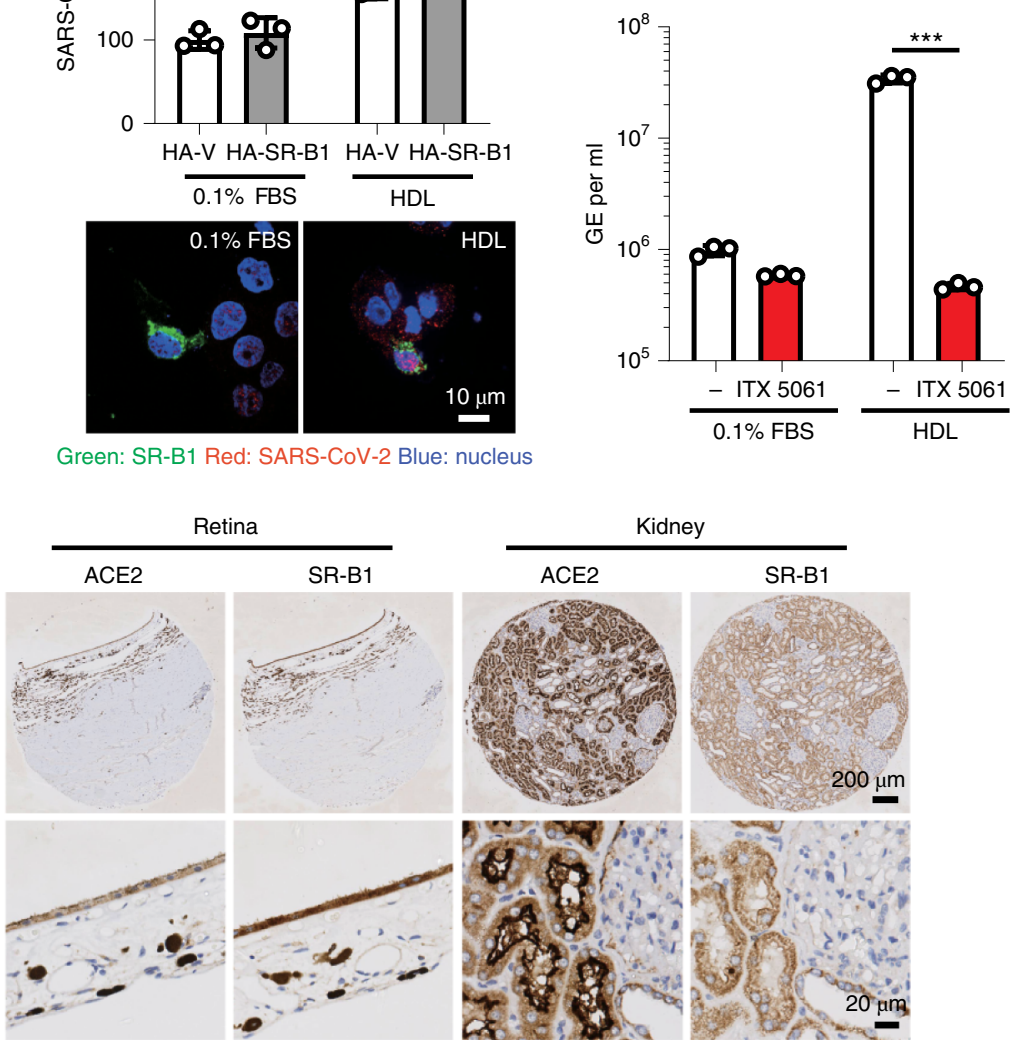

5061. ITX 5061 treatment strongly inhibited SARS-CoV-2 infection stimulated by HDL (Fig. 4g), demonstrating that SR-B1-mediated HDL uptake facilitates SARS-CoV-2 infection.

SARS-CoV-2 has been reported to attack multiple-organ systems and distribution analysis of SR-B1 in susceptible cells at the site of infection could shed new light on the tropism and potential transmission routes of the virus. Using immunohistochemistry, we found cell-surface SR-B1 staining in multiple regions of human lung tissue (Fig. 4h). Interestingly, coexpression of SR-B1 and ACE2 could also be seen in extrapulmonary tissues, including the colon, retina, small

intestine and testis (Fig. 4h and Extended Data Fig. 6a,b). Overall, our data demonstrate that SR-B1 is coexpressed with ACE2 in multiple susceptible tissues and could potentially be involved during SARS-CoV-2 infection.

\section{Discussion}

With over 30 million people infected, COVID-19 represents a major public health problem of high socioeconomic impact. However, treatment options for COVID-19 are limited, and a vaccine for prevention against COVID-19 infection is currently not available. 
Fig. 4 | SR-B1 mediates SARS-CoV-2 attachment and entry. a, 293T cells transfected with GFP-SR-B1 were exposed to SARS-CoV-2pp in solutions containing $6 \mu \mathrm{g} \mathrm{ml}^{-1} \mathrm{HDL}$ at $4^{\circ} \mathrm{C}$ for $30 \mathrm{~min}$ for attachment to the cell surface. All images were obtained by confocal microscopy. The scale bar indicates $10 \mu \mathrm{m}$. b. Huh-7 cells transfected with siSR-B1 were exposed to SARS-CoV-2 $(\mathrm{MOI}=0.1)$ in $10 \% \mathrm{FBS}$ at $4{ }^{\circ} \mathrm{C}$ for 10 min. All images were obtained by confocal microscopy. The scale bar indicates $40 \mu \mathrm{m}$. c,d, Huh-7 cells transfected with siSR-B1 (c) or HA-SR-B1 (d) were infected with SARS-CoV-2 for $1 \mathrm{~h}$, and the cells were collected $3 \mathrm{~h}$ after infection for detecting the gene copy number of the virus by RT-qPCR (upper graph) or intracellular SARS-CoV-2 staining by confocal microscopy using an anti-HA antibody (lower images). The scale bar indicates $40 \mu \mathrm{m}$ in $\mathbf{c}$ and $10 \mu \mathrm{m}$ in $\mathbf{d} . n=3$ independent biological experiments. $P=0.0002$ for HDL siCtrl versus HDL siSR-B1 (c), $P=0.0076$ for HDL HA-V versus HDL HA-SR-B1 by two-tailed Student's $t$-tests. e, For attachment, 293T cells transfected with HA-SR-B1 were exposed to SARS-2-S in solutions containing $0.1 \% \mathrm{FBS}$ or $6 \mu \mathrm{g} \mathrm{ml} \mathrm{l}^{-1} \mathrm{HDL}$ at $4^{\circ} \mathrm{C}$ for $30 \mathrm{~min}$. The cells were washed and detached before immunolabeling for flow cytometry. f, Huh-7 cells preincubated with ITX $5061(25 \mu \mathrm{M})$ or BLT-1 (25 $\mu$ M) were inoculated with SARS-CoV-2pp in solutions containing $6 \mu \mathrm{g} \mathrm{ml}^{-1} \mathrm{HDL}$, and pseudotyped viral entry was analysed by luciferase activity at $48 \mathrm{~h}$ after infection. Signals obtained without compounds in $0.1 \% \mathrm{FBS}$ were used for normalization. $n=3$ independent biological experiments. $P<0.0001 \mathrm{for} \mathrm{HDL}$ versus HDL ITX 5061, $P=0.0003$ for HDL versus HDL BLT-1 by one-way ANOVA and Bonferroni's post hoc analysis. $\mathbf{g}$, Huh-7 cells preincubated with ITX $5061(25 \mu \mathrm{M})$ were infected with SARS-CoV-2 $(\mathrm{MOI}=0.001)$ in solutions containing $6 \mu \mathrm{g} \mathrm{ml}^{-1} \mathrm{HDL}$ at the indicated concentrations for $1 \mathrm{~h}$, and the cells were collected to detect the gene copy number of the virus with RT-qPCR at $24 \mathrm{~h}$ after infection. $n=3$ independent biological experiments. $P<0.0001$ for HDL versus HDL ITX 5061 by two-tailed Student's t-tests. h, Representative images of immunohistochemical staining for ACE2 and SR-B1, which was performed on a paraffin-embedded human normal organ tissue microarray with antibodies against ACE2 or SR-B1, and counterstaining with hematoxylin was performed to show nuclei (blue). The scale bars indicate $200 \mu \mathrm{m}$ or $20 \mu \mathrm{m}$, as indicated. The results shown are representative of two independent biological experiments. The data are the mean \pm s.e.m. ${ }^{\star \star} P<0.01,{ }^{\star \star \star} P<0.001$.

The results of our study demonstrate that SR-B1 facilitates SARSCoV-2 cellular attachment, entry and infection. SARS-CoV-2 entry is inhibited by silencing SR-B1 expression and by SR-B1 antagonists. Moreover, SR-B1 expression levels have been shown to affect attachment, entry and replication of bona fide SARS-CoV-2 virus. Thus, as an entry cofactor, SR-B1 might represent a therapeutic target to limit SARS-CoV-2 infection.

$\mathrm{HDL}$ is an important lipoprotein present in human plasma that nonspecifically blocks or facilitates viral entry through SR-B1. In the present study, we observed that the presence of HDL significantly increases infection with SARS-CoV-2. Mechanistically, HDL facilitates viral attachment and entry, presumably by associating with SARS-2-S. An antibody blocking the putative cholesterol/ HDL-binding site on SARS-2-S1 was observed to strongly inhibit HDL-enhanced SARS-CoV-2pp entry. The SPR data do not provide sufficient evidence to claim that a stable interaction occurs between HDL and SARS-2-S1 protein. Future studies will need to explore the molecular nature of the interaction between HDL and SARS-2-S1 protein and the potential transfer of material derived from HDL to SARS-2-S1.

SR-B1 cannot bind to the SARS-2-S protein directly. However, HDL-enhanced SARS-2-S attachment and SARS-CoV-2 entry were greatly increased by SR-B1 expression. Importantly, SR-B1 expression conferred the greatest cell susceptibility to SARS-CoV2pp only when coexpressed with ACE2. Additional cell lines with varying degrees of SR-B1 and ACE2 receptor expression or primary targeted cells are needed to elucidate the molecular mechanism underlying how SR-B1 facilitates viral entry. Our present data support the notion that SR-B1 could function as a linker molecule that recruits viral particles to come into proximity with ACE2 by associating with HDL (Extended Data Fig. 7). An important step towards confirming such a model would be the identification of the SR-B1ACE2 membrane complex that is important for viral entry.

If SR-B1 acts as a cofactor defining SARS-CoV-2 attachment to target cells, an important question remains as to why the lipid transfer activity of SR-B1 promotes viral entry. We know that SR-B1-mediated lipid transfer from HDL results in local increases in the cholesterol content of cell membranes ${ }^{8,15}$. Cholesterol and the abundant presence of cholesterol within lipid rafts play essential roles in viral entry and fusion ${ }^{16-19}$, and cholesterol depletion from cellular membranes has been shown to inhibit SARS-CoV-2 infection $^{20}$. We thus speculate that SARS-CoV-2 may exploit the physiological function of SR-B1 to achieve its entry and fusion processes.

To understand how SR-B1 and ACE2 coordinate viral entry, it is important to compare receptor expression pattern(s) and localization in lower respiratory tissues. In the current study, we found that lower respiratory tissues express SR-B1 and ACE2, consistent with their ability to support SARS-CoV-2 entry. In addition, SR-B1 is coexpressed with ACE2 in multiple extrapulmonary tissues including the retina and testis. The existing expression profiles of SR-B1 and ACE2 also indicate their coexpression in multiple metabolic organs ${ }^{8,21-25}$, which could indicate an enhanced degree of trophism for extrapulmonary tissues, thereby contributing to the multiple-organ pathologies of COVID-19 (ref. ${ }^{26-30}$ ).

Cardiovascular disease and diabetes are associated with an increased risk of severe COVID-19. Approximately half of patients with COVID-19 have chronic underlying diseases, mainly cardiovascular and cerebrovascular diseases and diabetes ${ }^{31-33}$. In addition, increased COVID-19 mortality has been observed in patients with obesity or diabetes ${ }^{34}$. HDL-mediated cholesterol efflux and selective cholesterol transport are hallmarks of many chronic metabolic diseases $^{35,36}$. Although our current data did not suggest that HDL worsens COVID-19, future studies examining serum lipid levels in patients with pre-existing conditions, and exploring their possible clinical and mechanistic links, may uncover novel mechanisms of disease.

ACE2 has been identified as a functional SARS coronavirus receptor both in cell lines and in vivo ${ }^{37}$. S protein-mediated ACE2 downregulation then contributes to the severity of lung pathologies $^{38}$. Commonalities between SARS-CoV-2 and SARS-CoV infection suggest that antibodies, peptides and small compounds targeting ACE2 can be used in the treatment of SARS-CoV-2. Identifying SR-B1 as a SARS-CoV-2 entry cofactor, we propose that drugs that target SR-B1 could act as antivirals that limit SARS-CoV-2 infection. As a proof of concept, we have shown that ITX 5601, a clinically approved inhibitor of HCV infection, strongly inhibits SARS-CoV-2 infection of cultured cells. We propose that further testing of ITX 5601 in animal models of SARS-CoV-2 infection is warranted. In summary, our results show that SARS-CoV-2 virus can exploit SR-B1 function to promote its cellular entry and highlight potential antiviral approaches to interfere with viral entry.

\section{Methods}

Reagents and antibodies. His-tagged SARS-2-S (GenBank accession no. QHD43416.1; Val16-Pro1213; SPN-C52H9), SARS-2-S1 (GenBack accession no. QHD43416.1; Val16-Arg685; S1N-C52H3), SARS-2-S2 (GenBank accession no. QHD43416.1; Ser686-Pro1213; S2N-C52H5) and SARS-2-RBD (GenBank accession no. QHD43416.1; Arg319-Lys 537; SPD-C52H3) were obtained from Acro Biosystems. ITX 5061 (HY-19900) was from MedChemExpress. Cholesterol (C8667), campesterol (C5157), epicholesterol (R207349), BLT-1 (SML0059), HDL (L8039), DMEM (high glucose, D5796) and protease inhibitors (11206893001) 
were from Sigma-Aldrich. Human serum (A3969001) and FBS (A3160901) were from Gibco. Lipofectamine 2000 (11668-027) was from Invitrogen. A tissue microassay (MNO661) was from Biomax. The Monolith His-Tag Labeling kit (MO-L018) was from NanoTemper Technologies.

$\alpha$-Tubulin for immunoblotting (T9026, clone DM1A; 1:1,000 dilution), HRP-labelled anti-Flag (A8592, clone M2; 1:1,000 dilution), anti-human FITC-conjugated IgG (AP113F; 1:50 dilution) and anti-HA (H9658, clone HA-7; 1:20,000 dilution) were from Sigma-Aldrich. Antibodies against SR-B1 for immunoblotting (ab217318, clone EPR20190; 1:2,000 dilution) and immunohistochemistry (ab217318, clone EPR20190; 1:100 dilution) and antibodies against ACE2 for immunoblotting (ab108252, clone EPR4435(2); 1:1,000 dilution) and immunohistochemistry (ab108252, clone EPR4435(2); 1:50 dilution) were from Abcam. An antibody against ACE2 for FACS (21115-1-AP; 1:100 dilution) was from Proteintech. A PE anti-His antibody (362603, clone J095G46; 1:100 dilution) and an antibody against SR-B1 for FACS (363208, clone m1B9; dilution 1:100) were from BioLegend. The SARS-2-S mAb (GTX632604, clone 1A9; 1:1,000 dilution) was from GeneTex. The SARS-2-S1 antibody (40150-T62-COV2) for confocal microscopy was from Sino Biological. 1D2 mAb was from the laboratory of Wei Chen.

Plasmids. Expression plasmids for human HA-SR-B1 (H3784), Flag-ACE2 (H3673) and HA-ApoA1 (H3912) were obtained from Vigene Biosciences. Expression plasmids for SARS-2-S, VSV-G and the HIV-1 NL4-3 $\Delta$ Env Vpr Luciferase Reporter Vector (pNL4-3-Luc-R-E-) were obtained from the laboratory of Wei Chen. The codon-optimized (for human cells) SARS-2-S gene was based on GenBank ID QHD43416.1. All constructs were sequence verified. The siRNAs targeting human genes were obtained from Invitrogen and had the following target sequences:

siSR-B1-1 (860): 5'-CAAGUUCGGAUUAUUUGCUTT-3'

siSR-B1-2 (1007): 5'- CAUGAUCAAUGGAACUUCUTT-3'

siSR-B1-3 (1423): 5'-GCCUCUACAUGAAAUCUGUTT-3

Scrambled siRNA oligonucleotides from siSR-B1-3 were used as a control (siCtrl).

Cell culture and transfection. The 293T (CRL-3216), Vero E6 (CRL-1568), MDCK (CCL-34) and Hepa 1-6 (CRL-1830) cell lines were from the American Type Culture Collection. The Huh-7 (0403) cell line was from the Japanese Collection of Research Bioresources. The HeLa-hACE2 (012) cell line was from Beijing Vitalstar Biotechnology. All cell lines had been previously tested for mycoplasma contamination and were incubated in DMEM at $37^{\circ} \mathrm{C}$ under a humidified atmosphere with $5 \% \mathrm{CO}_{2}$. All media were supplemented with $10 \% \mathrm{FBS}, 100 \mathrm{U} \mathrm{ml}^{-1}$ penicillin, $0.1 \mathrm{mg} \mathrm{ml}^{-1}$ streptomycin, $1 \times$ nonessential amino acid solution and $10 \mathrm{mM}$ sodium pyruvate. Lipofectamine 2000 was used for transfection following the manufacturer's protocol.

MST. MST assay was conducted as previously described ${ }^{39}$. SARS-2-S-His-tagged, SARS-2-S1-His-tagged and SARS-2-S2-His-tagged proteins were labelled with NT-647 dye for $30 \mathrm{~min}$ at room temperature, as recommended by the Monolith His-Tag Labeling Kit RED-tris-NTA protocol (NanoTemper Technologies, MO-L008). The binding buffer used for reactions (50 mM Tris- $\mathrm{HCl}$ ( $\mathrm{pH} 7.5)$, $150 \mathrm{mM} \mathrm{NaCl}$ and $1 \mathrm{mM}$ dithiothreitol) contained micelles of $0.5 \%$ Fos-choline 13 , and a 16-step, twofold dilution curve of cholesterol and cholesterol derivatives ranged from $4 \mu \mathrm{M}$ to $122 \mathrm{pM}$. Cholesterol or other sterol dry powders were mixed with binding buffer at $25^{\circ} \mathrm{C}$ with mild agitation for $3 \mathrm{~h}$. Labelled protein $(100 \mathrm{nM}$, final concentration) in binding buffer was then added to a dilution curve of cholesterol or cholesterol derivatives at room temperature. Samples were loaded into standard glass capillaries (NanoTemper Technologies, MO-K022). MST was completed in three independent experiments on a Monolith NT.115 instrument (NanoTemper Technologies) running MO.Affinity Analysis (v.2.1.23333) with settings of $60-80 \%$ excitation power and $40 \%$ MST power at room temperature.

In the HDL-binding assay, labelled protein $(100 \mathrm{nM}$, final concentration) in binding buffer $(145 \mathrm{mM} \mathrm{NaCl})$ was added to a dilution curve of HDL at room temperature, where the dilution curve of HDL ranged from $6.25 \mu \mathrm{g} \mathrm{ml}^{-1}$ to $190.7 \mathrm{pg} \mathrm{m}^{-1}$ (16-step, twofold). Samples were loaded into standard glass capillaries (NanoTemper Technologies, MO-K022). MST was completed in three independent repeat experiments on a Monolith NT.115 instrument (NanoTemper Technologies) running MO.Control software with settings of $60-80 \%$ excitation power and $40 \%$ MST power at room temperature.

In the competition assay, $100 \mathrm{nM}$ labelled SARS-2-S in binding buffer was incubated with $200 \mathrm{nM}$ cholesterol either alone or with unlabelled SARS-2-S at the indicated concentrations.

Original data were obtained from MO.Affinity Analysis software. Fluorescence intensity values were averaged and expressed as the relative change over the results from the $0-\mu \mathrm{M}$ ligand condition. The data were fitted to saturation binding equations using GraphPad Prism 8.0. Dotted lines indicate where data could not be fitted.

SPR. SPR data were recorded on an OpenSPR-XT system (Nicoya Lifesciences) with OpenSPR software (3.11.6949.28944). For all experiments, $145 \mathrm{mM} \mathrm{NaCl}$ was used as the running buffer. His-tagged SARS-2-S1 or SARS-2-S2 was dissolved to a final concentration of $30 \mu \mathrm{g} \mathrm{ml}^{-1}$ in $145 \mathrm{mM} \mathrm{NaCl}$ before immobilization on NTA sensors (SEN-AU-100-3-NTA, Nicoya Lifesciences). Approximately 2,000 response units of protein were immobilized on the chip with a running buffer composed of $145 \mathrm{mM} \mathrm{NaCl}$. A series of HDL concentrations ranging from 0.313 to $10 \mu \mathrm{g} \mathrm{ml}^{-1}$ were tested at a flow rate of $20 \mu \mathrm{lmin}^{-1}$. The contact time was $300 \mathrm{~s}$ and the dissociation time was $300 \mathrm{~s}$. When the data collection was finished for each cycle, the sensor surface was regenerated with PBS buffer. The binding affinity between $\mathrm{HDL}$ and SARS-2-S1 or SARS-2-S2 was analysed at $25^{\circ} \mathrm{C}$. The binding curves were displayed using the steady-state affinity method incorporated in the Trace Drawer (v.1.8, Nicoya Lifesciences).

Peptide-cholesterol binding assay. The synthetic peptides listed below, encompassing the CARC-CRAC region of SARS-2-S, were purchased from Scilight-Peptide. A control peptide with a random amino acid sequence was also included in the assay.

Control (Ctrl): KKKARVRIFLYGFLLQLLMPVWTMKKK

24-42: KKKLPPAYTNSFTRGVYYPDKVKKK

129-150: KKKKVCEFQFCNDPFLGVYYHKNNKKKK

267-277: KKKVGYLQPRTFLLKKK

445-461: KKKVGGNYNYLYRLFRKSNLKKK

776-806: KKKKNTQEVFAQVKQIYKTPPIKDFGGFNFSQILKKK

1,203-1,218: KKKLGKYEQYIKWPWYIWLKKK

The N-terminal and C-terminal Lys residues increase the solubility of the hydrophobic CARC-CRAC transmembrane segment. Replacing the Pro residue located between the CARC and the CRAC with Trp conferred intrinsic fluorescence upon 280-nm excitation, with maximum emission at $350 \mathrm{~nm}$. Stock solutions of $5 \mathrm{mM}$ peptide in dimethylsulfoxide were diluted to a final concentration of $5 \mu \mathrm{M}$ in assay buffer containing $140 \mathrm{mM} \mathrm{NaCl}$ and $20 \mathrm{mM}$ Tris ( $\mathrm{pH}$ 8.5) supplemented with $2 \mathrm{mM}$ Tris(2-carboxyethyl)phosphine hydrochloride solution (TCEP) and 2\% (v/v) EtOH. Stock solutions of cholesterol in $\mathrm{EtOH}$ were diluted to final concentrations ranging between 0.5 and $50 \mu \mathrm{M}$ in $500-\mu$ l volumes of peptide solution, which were incubated overnight at $25^{\circ} \mathrm{C}$ with mild agitation. Fluorescence measurements were performed in triplicate on a Cary Eclipse fluorescence spectrophotometer (Agilent Technologies), with excitation and emission performed at 280 and $350 \mathrm{~nm}$, respectively. All measured fluorescence values fell within the linear range of the instrument. Fluorescence intensity values were averaged and expressed as relative change over the 0 - $\mu \mathrm{M}$ ligand condition. Data were fitted to saturation binding equations using GraphPad Prism v.8.0. Dotted lines indicate where data could not be fitted

Pseudovirus production. All pseudoparticles were generated in $293 \mathrm{~T}$ cells transfected with the HIV backbone vector pNL4-3.Luc.R-E together with the expression plasmids SARS-2-S or VSV-G. Pseudoparticle-containing supernatants were collected at $48 \mathrm{~h}$ and frozen to $-80^{\circ} \mathrm{C}$ after centrifugation at $2,000 \mathrm{~g}$ for $5 \mathrm{~min}$.

Pseudovirus entry assay. Cells were cultured in 96-well, transparent-bottom, flat culture plates (no. 3603, Corning) precoated with poly-D-lysine. To assess the inhibitory activity of chemicals, the indicated concentrations of chemicals were added to cells $4 \mathrm{~h}$ before infection. To determine the neutralization ability of antibodies, serially diluted antibodies were added to cells $1 \mathrm{~h}$ before infection. SARS-CoV-2pp preincubated with $\mathrm{HDL}$ at $25^{\circ} \mathrm{C}$ for $2 \mathrm{~h}$ was applied to the cells pretreated with chemicals or antibodies and infected at $37^{\circ} \mathrm{C}$ for $24 \mathrm{~h}$. After SARS-CoV-2pp infection, the efficiency of viral entry was determined through a firefly luciferase assay. Specifically, cells were washed with PBS once, and then $16 \mu \mathrm{l}$ of Cell Culture Lysis Reagent (E153A, Promega) was added to each well. Then, the plate was incubated for $15 \mathrm{~min}$ with rocking at room temperature, after which cell lysate $(8 \mu \mathrm{l})$ from each well was added to a 384 -well plate (no. 3574, Corning) followed by the addition of $16 \mu$ l of Luciferase Assay Substrate (no. E151A, Promega). Luciferase activity measurements were performed on a Spark $20 \mathrm{M}$ multimode microplate reader (Tecan) with Tecan Spark Control (v.2.1) software. All infection experiments were performed in a biosafety level-2 (BSL-2) laboratory. Cells without viruses, HDL and chemicals were used as blank controls, and cells without chemicals and HDL were used as viral controls. All the infection experiments were performed in a BSL-2 laboratory.

Western blot analysis and immunoprecipitation. Cells were lysed in NP40 cell lysis buffer with fresh protease inhibitors. Supernatants were separated by SDS-PAGE after centrifugation and transferred to polyvinylidene difluoride membranes for immunoblot analyses using the indicated primary antibodies: anti-Flag (1:5,000), anti-HA (1:5,000), anti-SR-B1 (1:3,000), anti-ACE2 $(1: 1,000)$ and anti- $\alpha$-Tubulin $(1: 8,000)$. An aliquot of the total lysate $(5 \%, v / v)$ was included as a control for the interaction assay and immunoprecipitation was performed with the indicated antibodies. Anti-rabbit or anti-mouse HRP-conjugated antibodies were then applied after three washes and the antigen-antibody complexes were visualized using chemiluminescence by a Tanon 5200 chemiluminescent imaging system, and analysed by Tanon Gelcap (v.5.22).

Infection with authentic SARS-CoV-2. The SARS-CoV-2 strain (BetaCoV/ Beijing/IME-BJ01/2020) used in the present study was originally isolated from a 
patient with COVID-19 returning from Wuhan, China with patient consent, and was stored at $-80^{\circ} \mathrm{C}$ in the State Key Laboratory of Pathogen and Biosecurity at Beijing Institute of Microbiology and Epidemiology ${ }^{40}$. All the experiments involving viruses were performed in the BSL-3 laboratory of the Beijing Institute of Microbiology and Epidemiology.

For experiments with HDL, SARS-CoV-2 isolate (MOI (multiplicity of infection) $=0.001$ ) was preincubated with the indicated concentrations of HDL at $37^{\circ} \mathrm{C}$ for $1 \mathrm{~h}$ before adding to the cells. After $1 \mathrm{~h}$ of infection, the above cells were washed three times with PBS before $200 \mu \mathrm{l}$ of DMEM was added. At $3 \mathrm{~h}$ or $24 \mathrm{~h}$ postinfection, cells were collected for viral RNA extraction with a viral RNA kit (52906, Qiagen) according to the manufacturer's instructions and detected by quantitative PCR with reverse transcription (RT-qPCR) assays with a One-Step PrimeScript RT-PCR kit (Takara, Japan) using SARS-CoV-2-specific primers on an Applied Biosystems 7500 Real-Time PCR System with QuantStudio Design \& Analysis Software (v.1.51).

For experiments involving chemicals and antibodies, cells were cultured in 96-well plates at $37^{\circ} \mathrm{C}$ overnight. The supernatant was discarded, and $100 \mu \mathrm{l}$ of medium containing different concentrations of drugs was added to the wells of the plates and incubated for $4 \mathrm{~h}$ (chemicals) or $1 \mathrm{~h}$ (for antibodies) before infection. The procedure above was repeated.

The TaqMan primers for SARS-CoV-2 were as follows:

5'-TCCTGGTGATTCTTCTTCAGG-3' and

\section{5'-TCTGAGAGAGGGTCAAGTGC- 3 '}

The SARS-CoV-2 probe used was as follows:

5'-FAM-AGCTGCAGCACCAGCTGTCCA-BHQ 1-3'.

Copies per millilitre were determined using a synthetic RNA fragment to amplify the target region.

Flow cytometry. For surface SR-B1 or ACE2 expression analysis, cells were washed with PBS two times and incubated with an APC-conjugated SR-B1 (1:100) or anti-ACE2 (1:100) antibody, followed by a secondary donkey anti-rabbit antibody (1:200) conjugated to Alexa Fluor 488. Measurement of cellular fluorescence was determined by FACS. Thirty thousand events were registered for each experiment. Cellular fluorescence was quantitated as the mean fluorescence intensity or the percentage of positive cells.

For SARS-2-S cell-surface binding, SARS-2-S proteins (140 nM) and HDL $\left(6 \mu \mathrm{g} \mathrm{ml}^{-1}\right)$ preincubated at $37^{\circ} \mathrm{C}$ for $1 \mathrm{~h}$ were then added to the cells. After incubating at $4^{\circ} \mathrm{C}$ for $30 \mathrm{~min}$, the cells were washed two times with PBS containing $2 \% \mathrm{FBS}$ and then incubated for $30 \mathrm{~min}$ at room temperature with a PE-conjugated antibody (PE anti-His tag). Then, the cells were washed again with PBS containing $2 \%$ FBS, after which binding was detected by flow cytometry. The cells were analysed on an Invitrogen Attune NxT Acoustic Focusing cytometer. Attune NxT Software (v.3.1.1243.0) and FlowJo v.10.0.7 software were used to process the data based on proper gating strategy (Extended Data Fig. 2c). At least 10,000 cells were analysed for each sample.

Confocal microscopy. Cells grown on poly-L-lysine-coated coverslips were fixed in $4 \%$ PFA for $10 \mathrm{~min}$ and then washed three times with PBS. For the viral entry assay, cells were permeabilized in $0.2 \%$ Triton X-100 in PBS and blocked with $2 \%$ BSA in PBS for $1 \mathrm{~h}$. Nonpermeabilized cells were used for viral attachment assay. A SARS-2-S1 antibody and Alexa Fluor 555-labelled donkey anti-rabbit IgG $(\mathrm{H}+\mathrm{L})$ were used for immunofluorescence staining. Nuclear DNA staining was performed with DAPI $\left(0.5 \mu \mathrm{g} \mathrm{ml}^{-1}\right.$, Sigma-Aldrich, D9542). Fluorescent images were acquired under a Nikon A1 confocal microscope using Nikon A1 Elements software (v.4.20). The images were processed using Volocity (v.6.1.1, PerkinElmer). Viral attachment to or entry into cells was quantified using an NIS-Elements Advanced Research Imaging software to measure the relative fluorescence intensity. The DAPI channel was used to count the number of cells per image. Fluorescence was divided by cell count to calculate the fluorescence per cell in each condition.

Immunohistochemistry. Immunohistochemical staining for ACE2 and SR-B1 was performed on the paraffin-embedded human normal organ tissue microarray (MNO661, Biomax). Antigen retrieval was performed by placing the slides in $10 \mathrm{mM}$ sodium citrate buffer ( $\mathrm{pH}$ 6.0) and maintaining at a sub-boiling temperature for $10 \mathrm{~min}$. After blocking in $1 \%$ normal goat serum, the sections were incubated with ACE2 or SR-B1 monoclonal antibodies at $4^{\circ} \mathrm{C}$ overnight in a humidified chamber, which was followed by an incubation with HRP-labelled goat anti-mouse IgG secondary antibody (Beijing ZSGB Biotechnology, ZDR-5307). Subsequently, the sections were incubated with a goat anti-rabbit IgG secondary antibody (HRP) (Beijing ZSGB Biotechnology, PV9001) for $60 \mathrm{~min}$ and then visualized with 3,30-diaminobenzidine tetrahydrochloride (DAB). The slices were viewed under an Olympus microscope.

Statistical methods. In the present study, GraphPad Prism 8.0 was used for statistical calculations and data plotting. Differences between two independent samples were evaluated by two-tailed Student's $t$-tests. Differences between multiple samples were analysed by one-way analysis of variance (ANOVA) followed by Bonferroni's post hoc analysis. The correlation between SR-B1 expression and the efficiency of viral entry was determined by Spearman's nonparametric test.
All tests were two-tailed, unless otherwise indicated. We considered $P \leq 0.05$ to be statistically significant. Significance values were set as follows: NS (not significant), $P>0.05 ;{ }^{*} P<0.05 ;{ }^{*} P<0.01 ;{ }^{* *} P<0.001$. Further information on the statistics methods used in this paper can be found in the 'Statistics and Reproducibility' file in the Supplementary Information.

Reporting Summary. Further information on research design is available in the Nature Research Reporting Summary linked to this article.

\section{Data availability}

The data that support the findings of this study are available from the corresponding author upon reasonable request. Source data are provided with this paper.

Received: 3 June 2020; Accepted: 12 November 2020;

Published online: 26 November 2020

\section{References}

1. World Health Organization. Coronavirus Disease (COVID-19) Pandemic. https://www.who.int/emergencies/diseases/novel-coronavirus-2019 (2020).

2. Pelkmans, L. \& Helenius, A. Insider information: what viruses tell us about endocytosis. Curr. Opin. Cell Biol. 15, 414-422 (2003).

3. Wang, H. et al. SARS coronavirus entry into host cells through a novel clathrin- and caveolae-independent endocytic pathway. Cell Res. 18, 290-301 (2008)

4. Walls, A. C. et al. Structure, function, and antigenicity of the SARS-CoV-2 spike glycoprotein. Cell 181, 281-292.e286 (2020).

5. Hoffmann, M. et al. SARS-CoV-2 cell entry depends on ACE2 and TMPRSS2 and is blocked by a clinically proven protease inhibitor. Cell 181, 271-280.e8 (2020).

6. Chi, X. et al. A neutralizing human antibody binds to the N-terminal domain of the Spike protein of SARS-CoV-2. Science 369, 650-655 (2020).

7. Shen, W. J., Asthana, S., Kraemer, F. B. \& Azhar, S. Scavenger receptor B type 1: expression, molecular regulation, and cholesterol transport function. J. Lipid Res. 59, 1114-1131 (2018).

8. Shen, W. J., Azhar, S. \& Kraemer, F. B. SR-B1: a unique multifunctional receptor for cholesterol influx and efflux. Annu. Rev. Physiol. 80, 95-116 (2018).

9. Kolleck, I., Sinha, P. \& Rustow, B. Vitamin E as an antioxidant of the lung: mechanisms of vitamin E delivery to alveolar type II cells. Am. J. Respir. Crit. Care Med. 166, S62-S66 (2002).

10. Kolleck, I. et al. HDL is the major source of vitamin E for type II pneumocytes. Free Radic. Biol. Med. 27, 882-890 (1999).

11. Catanese, M. T. et al. Role of scavenger receptor class B type I in hepatitis C virus entry: kinetics and molecular determinants. J. Virol. 84, 34-43 (2010).

12. Fantini, J., Di Scala, C., Baier, C. J. \& Barrantes, F. J. Molecular mechanisms of protein-cholesterol interactions in plasma membranes: functional distinction between topological (tilted) and consensus (CARC/CRAC) domains. Chem. Phys. Lipids 199, 52-60 (2016).

13. Masson, D. et al. Increased HDL cholesterol and apoA-I in humans and mice treated with a novel SR-BI inhibitor. Arterioscler. Thromb. Vasc. Biol. 29, 2054-2060 (2009).

14. Raldua, D. \& Babin, P. J. BLT-1, a specific inhibitor of the HDL receptor SR-BI, induces a copper-dependent phenotype during zebrafish development. Toxicol. Lett. 175, 1-7 (2007).

15. Sorci-Thomas, M. G. \& Thomas, M. J. High density lipoprotein biogenesis, cholesterol efflux, and immune cell function. Arterioscler. Thromb. Vasc. Biol. 32, 2561-2565 (2012).

16. Bajimaya, S., Frankl, T., Hayashi, T. \& Takimoto, T. Cholesterol is required for stability and infectivity of influenza A and respiratory syncytial viruses. Virology 510, 234-241 (2017).

17. Osuna-Ramos, J. F., Reyes-Ruiz, J. M. \& Del Angel, R. M. The role of host cholesterol during flavivirus infection. Front. Cell. Infect. Microbiol. 8, 388 (2018).

18. Dou, X. et al. Cholesterol of lipid rafts is a key determinant for entry and post-entry control of porcine rotavirus infection. BMC Vet. Res. 14, 45 (2018).

19. Li, G. M., Li, Y. G., Yamate, M., Li, S. M. \& Ikuta, K. Lipid rafts play an important role in the early stage of severe acute respiratory syndrome-coronavirus life cycle. Microbes Infect. 9, 96-102 (2007).

20. Radenkovic, D., Chawla, S., Pirro, M., Sahebkar, A. \& Banach, M. Cholesterol in relation to COVID-19: should we care about it? J. Clin. Med. 9, 1909 (2020)

21. Julovi, S. M. et al. Apolipoprotein A-II plus lipid emulsion enhance cell growth via SR-B1 and target pancreatic cancer in vitro and in vivo. PLoS ONE 11, e0151475 (2016).

22. Johnson, A. C. M., Yabu, J. M., Hanson, S., Shah, V. O. \& Zager, R. A Experimental glomerulopathy alters renal cortical cholesterol, SR-B1, ABCA1, and HMG CoA reductase expression. Am. J. Pathol. 162, 283-291 (2003). 
23. Hardenberg, J. B. \& Luft, F. C. Covid-19, ACE2 and the kidney. Acta Physiol. (Oxf.) 230, e13539 (2020).

24. Zhang, H. et al. Specific ACE2 expression in small intestinal enterocytes may cause gastrointestinal symptoms and injury after 2019-nCoV infection. Int. J. Infect. Dis. 96, 19-24 (2020).

25. Liu, F. et al. ACE2 expression in pancreas may cause pancreatic damage after SARS-CoV-2 infection. Clin. Gastroenterol. Hepatol. 18, 2128-2130 e2122 (2020).

26. Seah, I. \& Agrawal, R. Can the coronavirus disease 2019 (COVID-19) affect the eyes? A review of coronaviruses and ocular implications in humans and animals. Ocul. Immunol. Inflamm. 28, 391-395 (2020).

27. Hollstein, T. et al. Autoantibody-negative insulin-dependent diabetes mellitus after SARS-CoV-2 infection: a case report. Nat. Metab. 2, 1021-1024 (2020)

28. Cheng, Y. et al. Kidney disease is associated with in-hospital death of patients with COVID-19. Kidney Int. 97, 829-838 (2020).

29. Zhang, C., Shi, L. \& Wang, F. S. Liver injury in COVID-19: management and challenges. Lancet Gastroenterol. Hepatol. 5, 428-430 (2020).

30. Zhang, H. et al. Digestive system is a potential route of COVID-19: an analysis of single-cell coexpression pattern of key proteins in viral entry process. Gut 69, 1010-1018 (2020).

31. Lai, C. C., Shih, T. P., Ko, W. C., Tang, H. J. \& Hsueh, P. R. Severe acute respiratory syndrome coronavirus 2 (SARS-CoV-2) and coronavirus disease-2019 (COVID-19): the epidemic and the challenges. Int. J. Antimicrob. Agents 55, 105924 (2020)

32. Bajgain, K. T., Badal, S., Bajgain, B. B. \& Santana, M. J. Prevalence of comorbidities among individuals with COVID-19: a rapid review of current literature. Am. J. Infect. Control https://doi.org/10.1016/j.ajic.2020.06.213 (2020).

33. Chen, N. et al. Epidemiological and clinical characteristics of 99 cases of 2019 novel coronavirus pneumonia in Wuhan, China: a descriptive study. Lancet 395, 507-513 (2020)

34. Ceriello, A. et al. Issues of cardiovascular risk management in people with diabetes in the COVID-19 era. Diabetes Care 43, 1427-1432 (2020).

35. Assmann, G. \& Gotto, A. M. Jr. HDL cholesterol and protective factors in atherosclerosis. Circulation 109, III8-III14 (2004).

36. Rashid, S. \& Genest, J. Effect of obesity on high-density lipoprotein metabolism. Obes. (Silver Spring) 15, 2875-2888 (2007).

37. $\mathrm{Li}, \mathrm{W}$. et al. Angiotensin-converting enzyme 2 is a functional receptor for the SARS coronavirus. Nature 426, 450-454 (2003).

38. Glowacka, I. et al. Differential downregulation of ACE2 by the spike proteins of severe acute respiratory syndrome coronavirus and human coronavirus NL63. J. Virol. 84, 1198-1205 (2010).
39. Jerabek-Willemsen, M., Wienken, C. J., Braun, D., Baaske, P. \& Duhr, S Molecular interaction studies using microscale thermophoresis. Assay Drug Dev. Technol. 9, 342-353 (2011).

40. Zhang, N. N. et al. A thermostable mRNA vaccine against COVID-19. Cell 182, 1271-1283.e1216 (2020).

\section{Acknowledgements}

We thank the National Key Research and Development Programme of China (grant no. 2018YFA0900800), the National Natural Science Foundation of China (grant nos. $81671973,31670761,31872715,81773205$ and 81572620), the Beijing Natural Science Foundation (grant nos. 5182029 and 5182030) and the Shandong Provincial Natural Science Foundation of China (grant no. ZR2015HM003) for their support. We also thank Q. Chang for the MST data collection at the Protein Preparation and Characterization Platform of the Tsinghua University Technology Centre for Protein Research. Extended Data Fig. 7 was created and exported with BioRender.com under a paid subscription.

\section{Author contributions}

C.W., L.W., Q.Y., Xiaolin Wang, J.Z., Xiaopan Yang, Y. Zhang, J.S., J.G., H.Y., H. Li, W.Z., N.W., Y.P., Y.D., D.L., H. Lin and J.F. performed the experiments. C.F., Xiaoli Yang, Y.W., Xuejun Wang, J.L., Z.Z., R.W., P.D., Y. Zong and F.Y. provided reagents and intellectual input. C.Q., C.W., R.Z., W.C., Q.G., Y.C. and H.Z. designed and coordinated the study and wrote the manuscript. All authors read and approved the manuscript.

\section{Competing interests}

The authors declare no competing interests.

\section{Additional information}

Extended data is available for this paper at https://doi.org/10.1038/s42255-020-00324-0. Supplementary information is available for this paper at https://doi.org/10.1038/ s42255-020-00324-0.

Correspondence and requests for materials should be addressed to R.Z., Y.C. or H.Z.

Peer review information Nature Metabolism thanks the anonymous reviewers for their contribution to the peer review of this work. Primary Handling Editor: George Caputa.

Reprints and permissions information is available at www.nature.com/reprints.

Publisher's note Springer Nature remains neutral with regard to jurisdictional claims in published maps and institutional affiliations.

(c) The Author(s), under exclusive licence to Springer Nature Limited 2020 
a

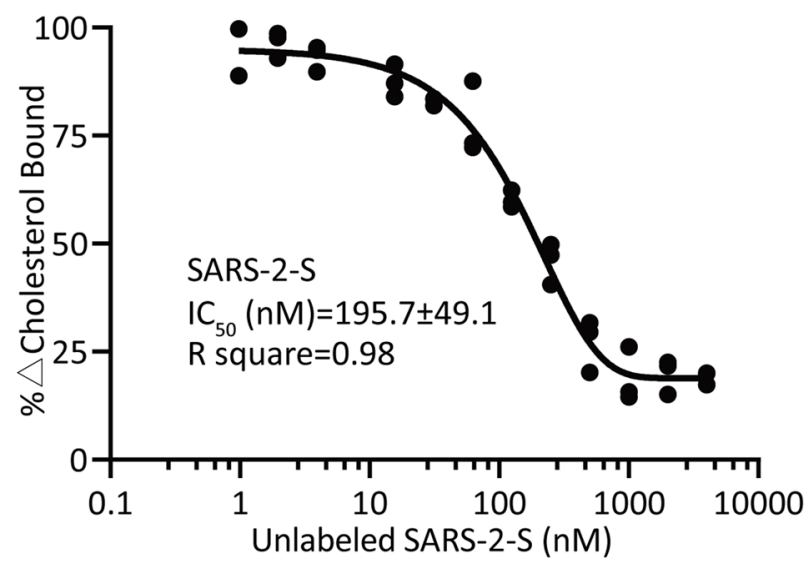

C

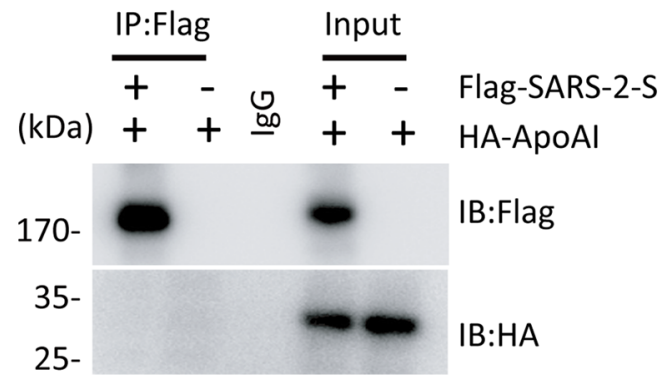

e

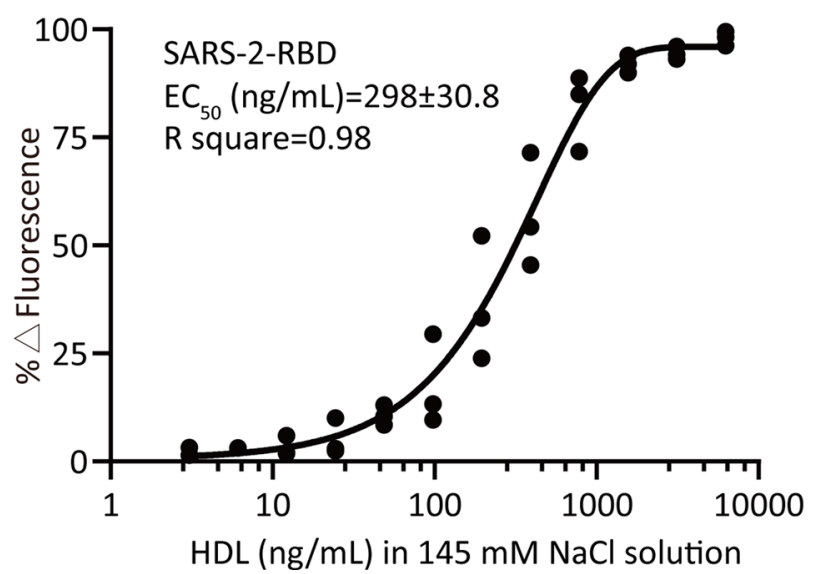

b

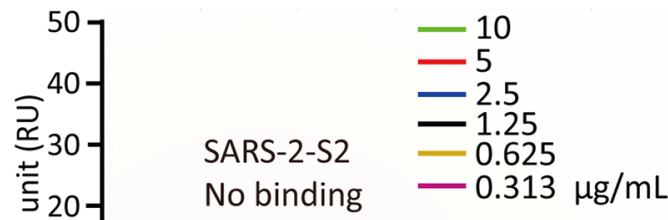

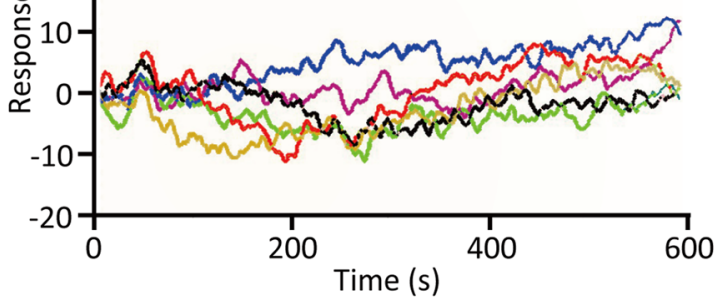

d

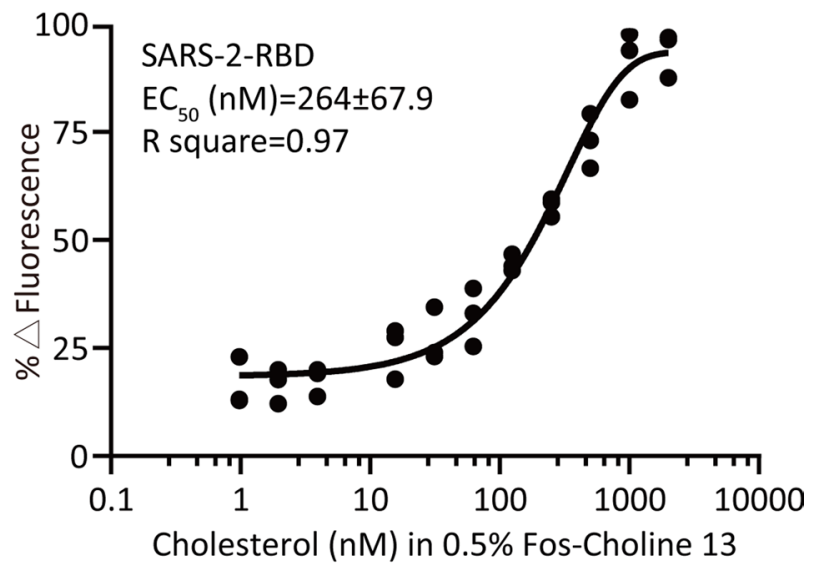

Extended Data Fig. 1 | SARS-2-S binds to cholesterol and HDL. a, Competitive binding of the indicated concentrations of unlabeled SARS-2-S with NT-647-NHS dye-labeled SARS-2-S in 0.5\% fos-choline 13 micelles containing $200 \mathrm{nM}$ cholesterol. The IC ${ }_{50}$ was determined by the Hill slope. $\mathrm{n}=3$ independent biological experiments. $\mathbf{b}$, Kinetic profile of interactions between SARS-2-S2 and HDL by SPR analysis. c, Immunoprecipitation analysis in 293T cells transfected with Flag-SARS-2-S and HA-ApoA1. The approximate molecular weight ( $\mathrm{kDa}$ ) marker positions are indicated to the left of the blot. d, e, MST analysis of interactions between SARS-2-RBD and cholesterol (d) or HDL (e). The data were derived from the effects of cholesterol or HDL on the fluorescence decay of fluorescently labeled proteins. The $\mathrm{EC}_{50}$ was determined by the Hill slope. $\mathrm{n}=3$ independent biological experiments. 
a

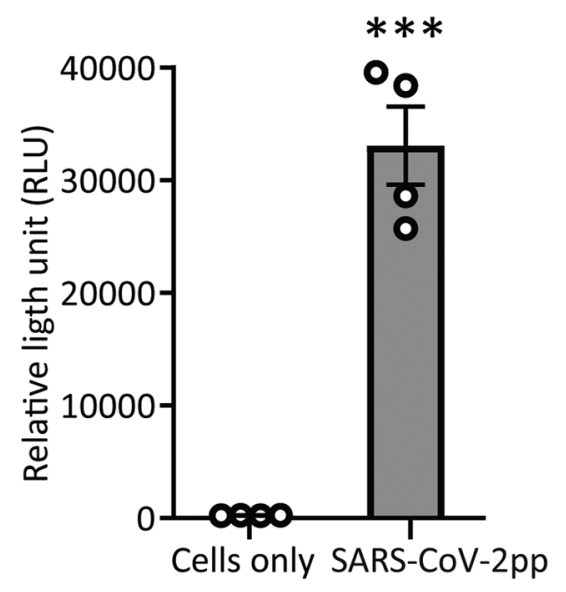

C

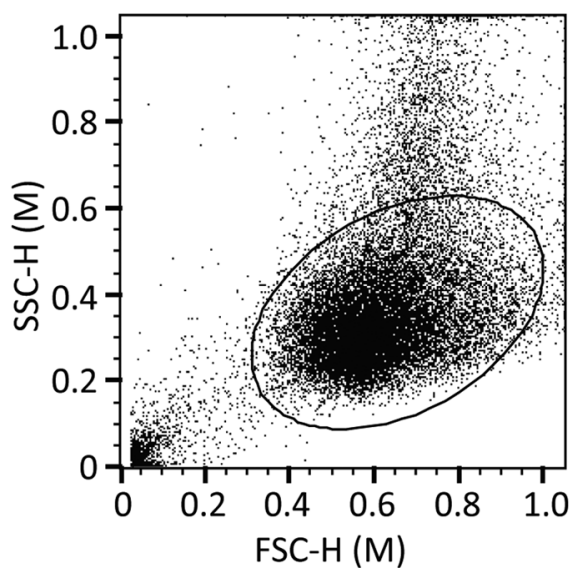

b

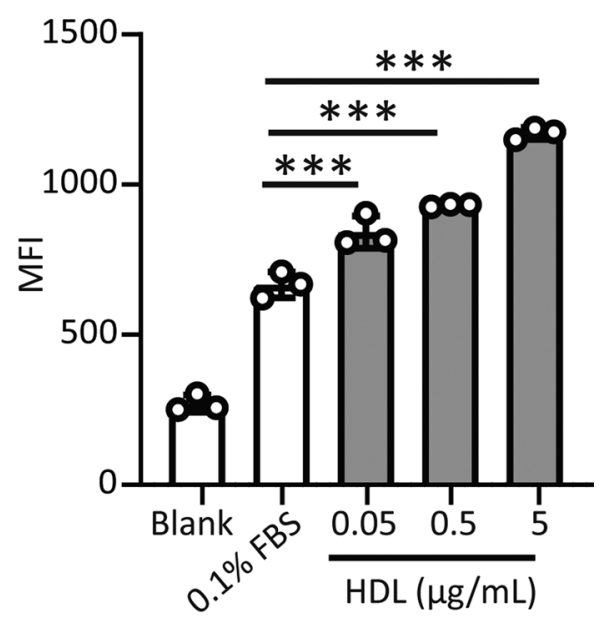

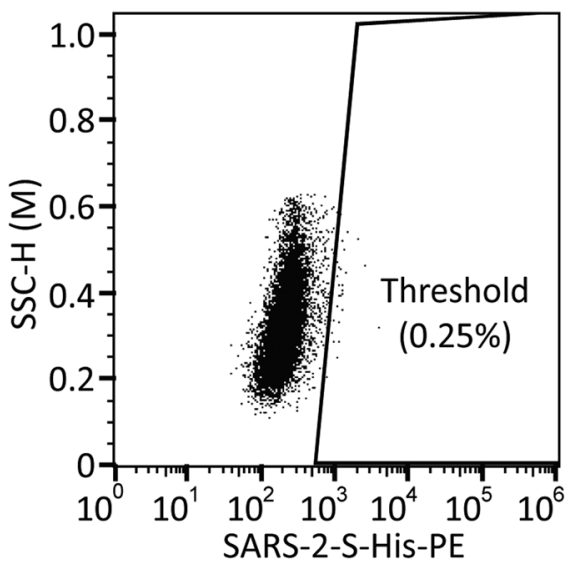

Extended Data Fig. 2 | SARS-2-S-HDL binding enhances SARS-CoV-2 entry. a, Huh-7 cells were inoculated with SARS-CoV-2pp in 10\% FBS, and pseudotyped viral entry was analyzed by luciferase activity at $48 \mathrm{~h}$ after infection. $\mathrm{n}=4$ independent biological experiments. $\mathrm{P}<0.0001 \mathrm{for} C$ ells only vs SARS-CoV-2pp by two-tailed Student's t-tests. b, Flow cytometry analysis of SARS-2-S adhesion to HeLa-hACE2 cells. The MFI was quantified from Fig. 2f. $\mathrm{n}=3$ independent biological experiments. $\mathrm{P}=0.0002$ for $0.1 \% \mathrm{FBS}$ vs $\mathrm{HDL}(0.05 \mu \mathrm{g} / \mathrm{mL}), \mathrm{P}<0.0001 \mathrm{for} 0.1 \% \mathrm{FBS}$ vs HDL $(0.5 \mu \mathrm{g} / \mathrm{mL}), \mathrm{P}<0.0001$ for $0.1 \%$ FBS vs HDL $(5 \mu \mathrm{g} / \mathrm{mL})$ by one-way ANOVA and Bonferroni's post hoc analysis. c, Gating strategy of flow cytometry. Cells were first gated on the basis of their scatter properties and single cells were identified using FSC-H vs. FSC-W. Spike-His-PE positive cells were then determined from a negative control with a threshold of $0.25 \%$. The data are the mean \pm SEM. ${ }^{\star \star \star} P<0.001$ by two-tailed Student's t-tests. 
a
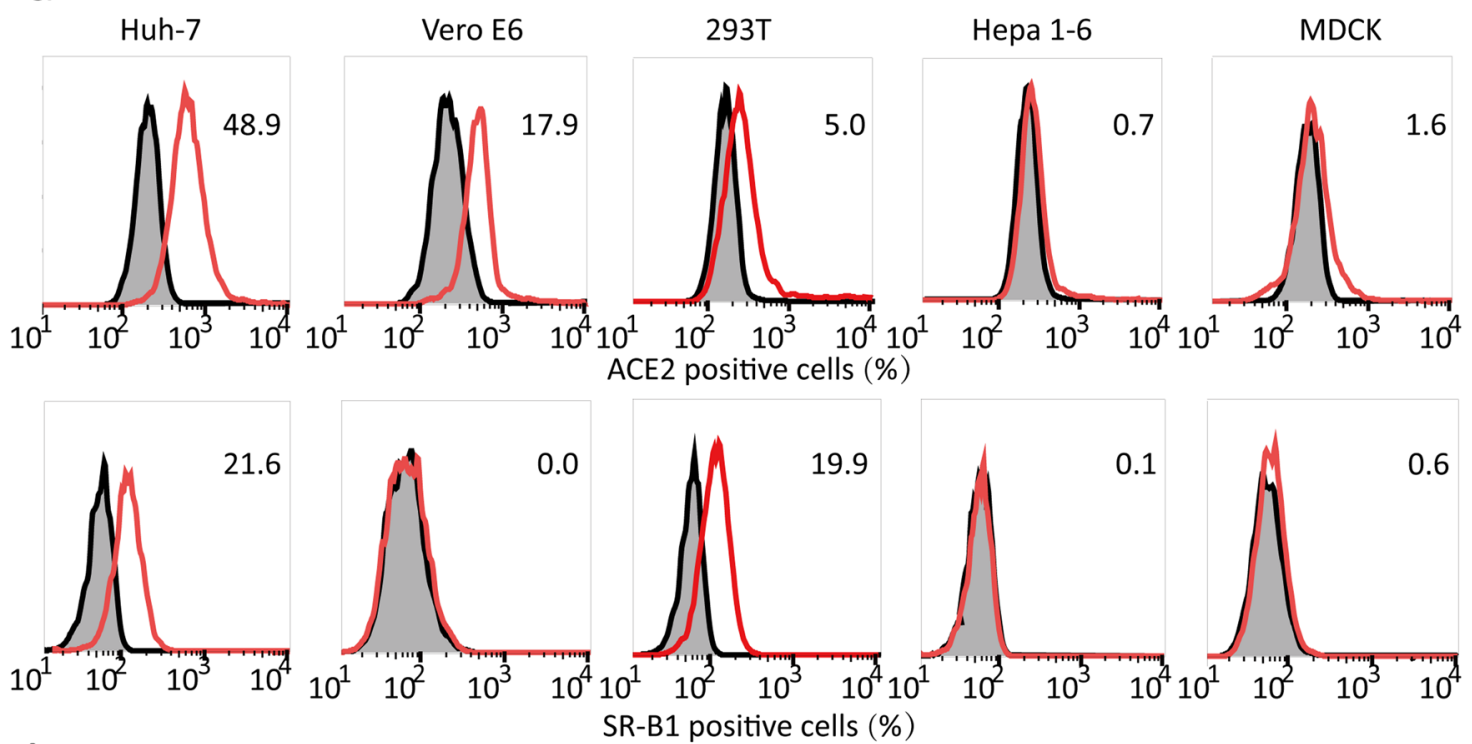

b
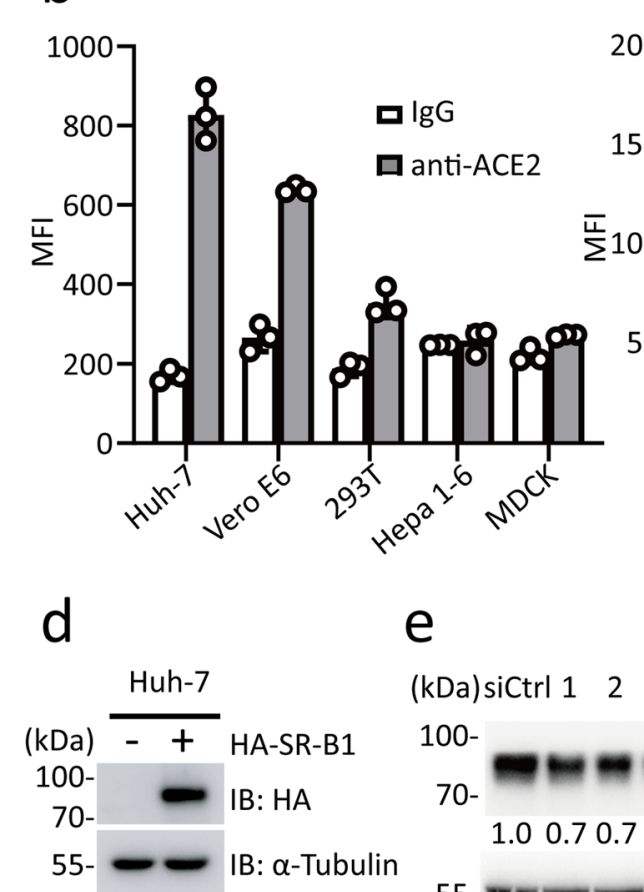

e

(kDa)sict

70-

$1.00 .70 .70 .4 \begin{aligned} & \text { Relative } \\ & \text { expressions }\end{aligned}$

55- - - IB: $\alpha$-Tubulin g

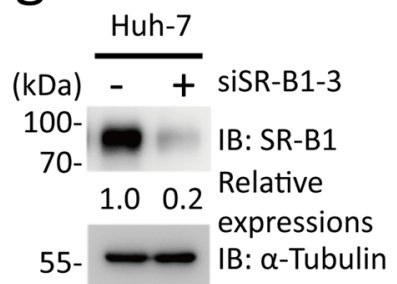

C

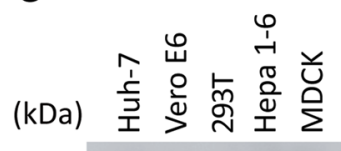
口anti-SR-B1 $130-\quad m-$ IB: ACE2 $100-$ $100-$

$70-$

55- - - - IB: $\alpha$-Tubulin

f siSR-B1 oligos

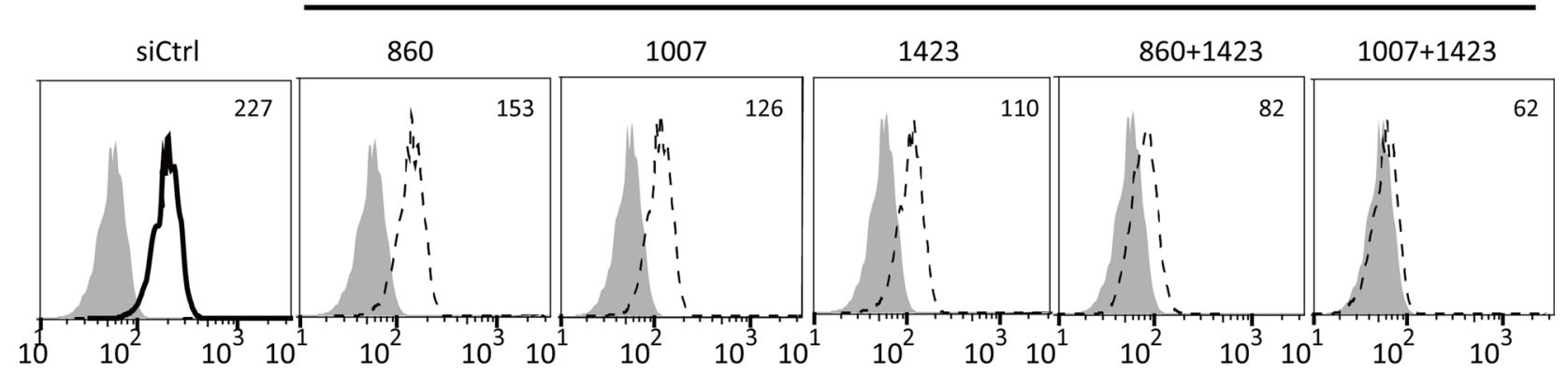

Extended Data Fig. 3 | See next page for caption. 
Extended Data Fig. 3 | Analysis and modulation of SR-B1 expression in multiple human and animal cell lines. a, b, c, Flow cytometry (a-b) or immunoblot (c) analysis of ACE2 and SR-B1 expression in the indicated cell lines. $n=3$ independent biological experiments (b). d, e, Immunoblot analysis of SR-B1 expression in Huh-7 cells transfected with HA-SR-B1 from Fig. 3a (d) or transfected with three siRNA oligos for SR-B1 from Fig. 3b (e). $\alpha$-Tubulin was used as the equal loading control. No. 3 oligos (1423; siSR-B1-3) were used for the pseudotyped viral infection assay. f, Flow cytometry analysis of SR-B1 expression in Huh-7 cells transfected with three siRNA oligos for SR-B1 in different combinations. g, h, Immunoblot analysis of SR-B1 expression in Huh-7 cells transfected with siSR-B1-3 from Fig. 3d (g) or Vero E6 cells transfected with HA-SR-B1 from Fig. 3e (h). $\alpha$-Tubulin was used as the equal loading control. The results shown are representative of three independent experiments. The data are the mean \pm SEM. 
a

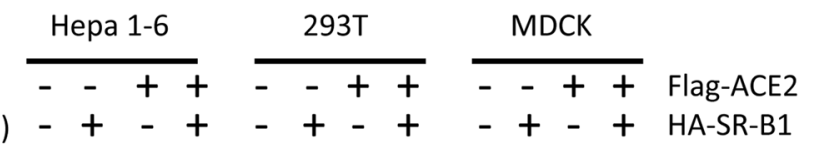

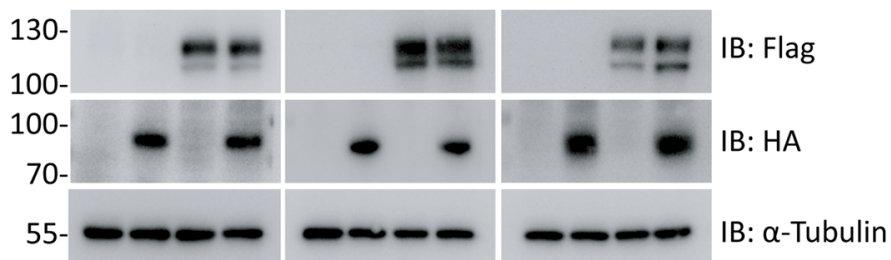

b

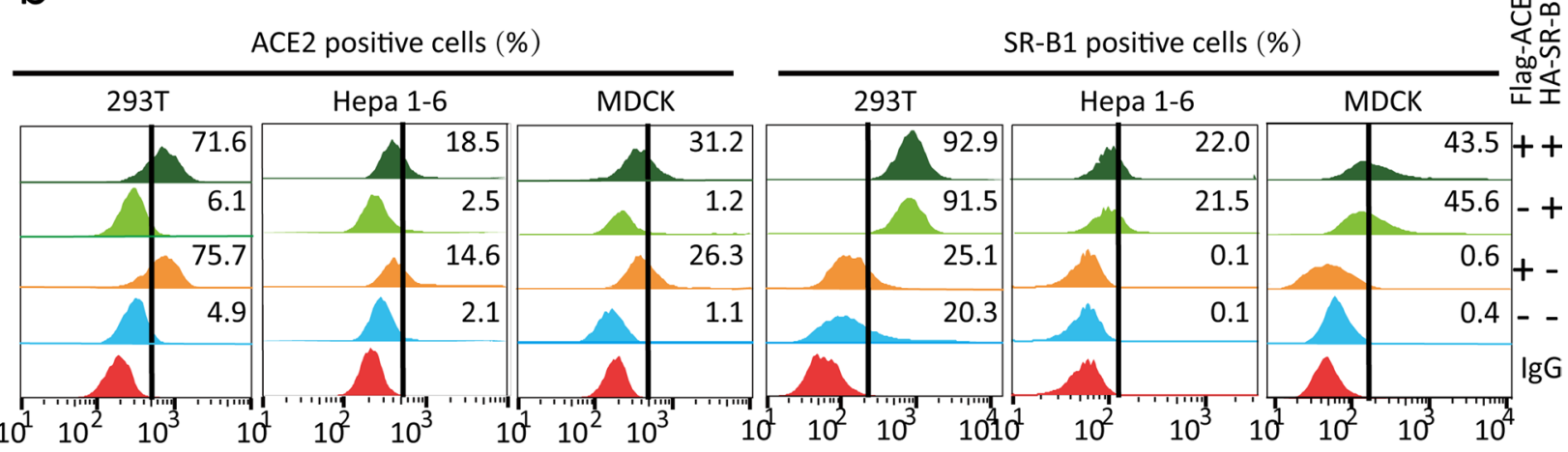

C
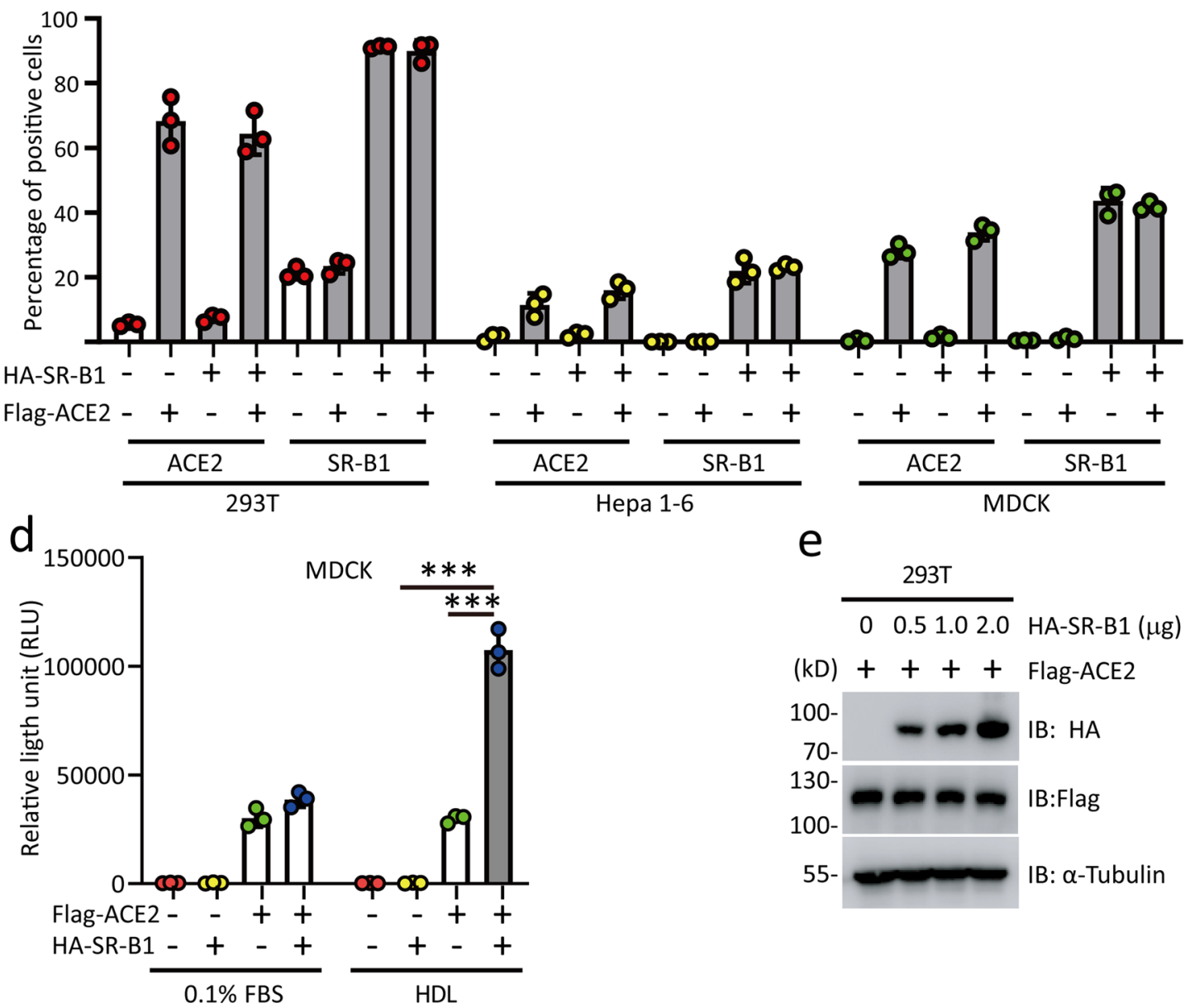

e

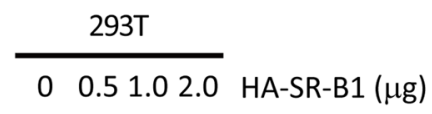

$(\mathrm{kD})++++$ Flag-ACE2

100-

70-

130

100

$-\infty-\infty$ IB:Flag

55

IB: $\alpha$-Tubulin

\begin{tabular}{lllllll} 
Flag-ACE2 - & - & + & + & - & - & + \\
HA-SR-B1 & + & - & + & - & + & + \\
\hline
\end{tabular}

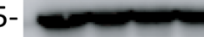

IB: HA

$-\infty$

Extended Data Fig. 4 | See next page for caption. 
Extended Data Fig. 4 | SR-B1 expression confers susceptibility to SARS-CoV-2 infection. a, b, c, Immunoblot analysis (a) or flow cytometry analysis (b-c) of ACE2 and SR-B1 expression in the indicated cell lines transfected with plasmids encoding ACE2 and or SR-B1 from Fig. 3f, $g$ and Extended Data Fig. 4d. $\mathrm{n}=3$ independent biological experiments (c). d, MDCK cells were transfected with plasmids encoding ACE2, SR-B1 or both and then challenged with SARS-CoV-2pp in solutions containing $0.1 \%$ FBS or $6 \mu \mathrm{g} / \mathrm{mL} \mathrm{HDL} . \mathrm{n}=3$ independent biological experiments. $\mathrm{P}<0.0001$ for HDL SR-B1 vs HDL ACE2 + $\mathrm{SR}-\mathrm{B} 1, \mathrm{P}=0.0001$ for HDL ACE2 vs HDL ACE2 + SR-B1 by one-way ANOVA and Bonferroni's post hoc analysis. e, Immunoblot analysis of ACE2 and SR-B1 expression in 293 T cells transfected with Flag-ACE2 together with increasing concentrations of HA-SR-B1 from Fig. 3h. The data are the mean \pm SEM. $\star \star \star P<0.001$ 
a

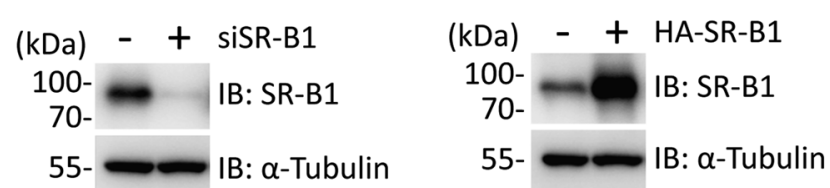

C

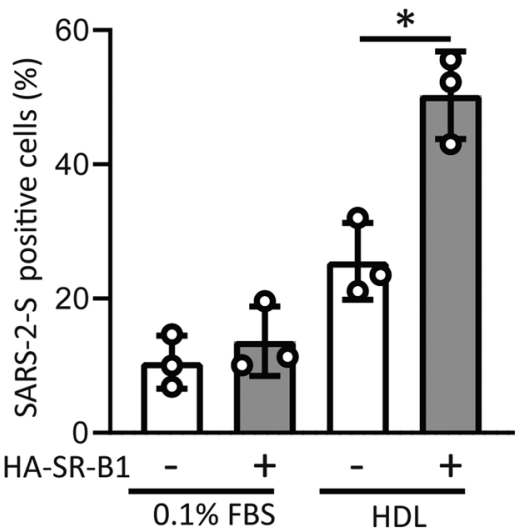

f

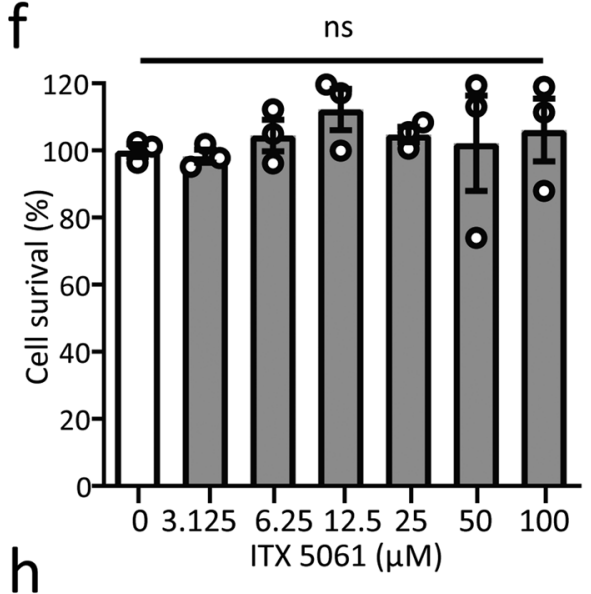

$\mathrm{h}$

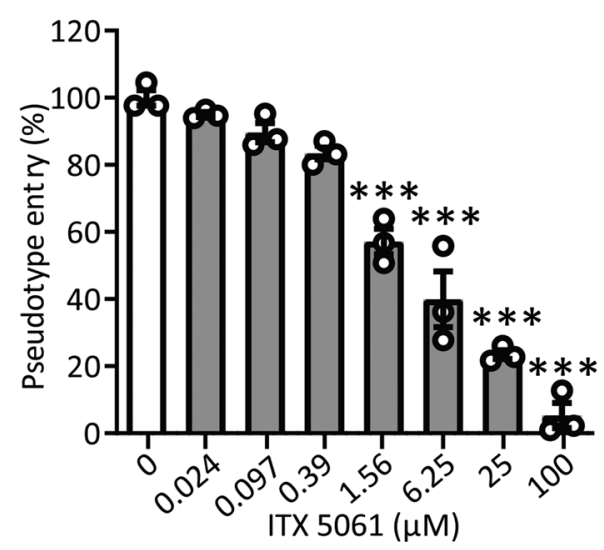

b

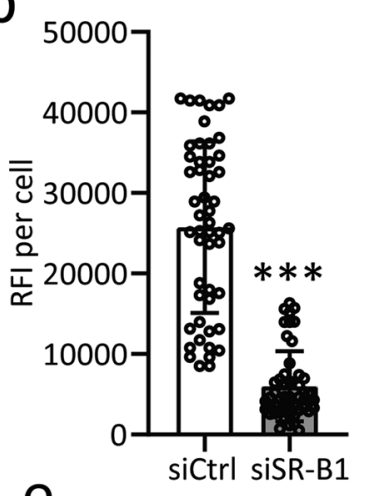

e

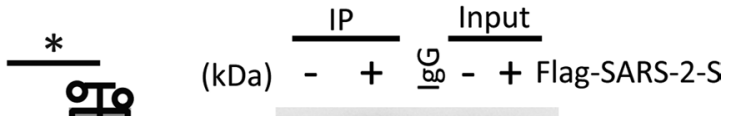

IB: ACE2

IB: SR-B1

IB: Flag

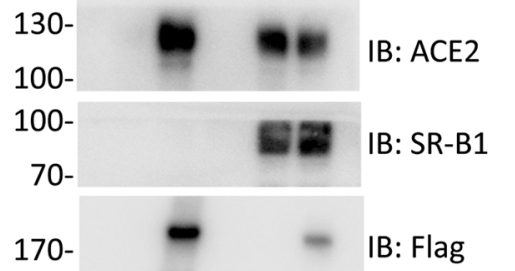

g

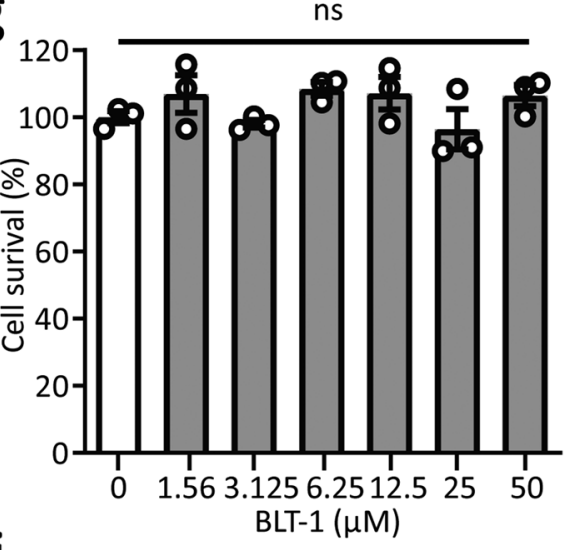

i

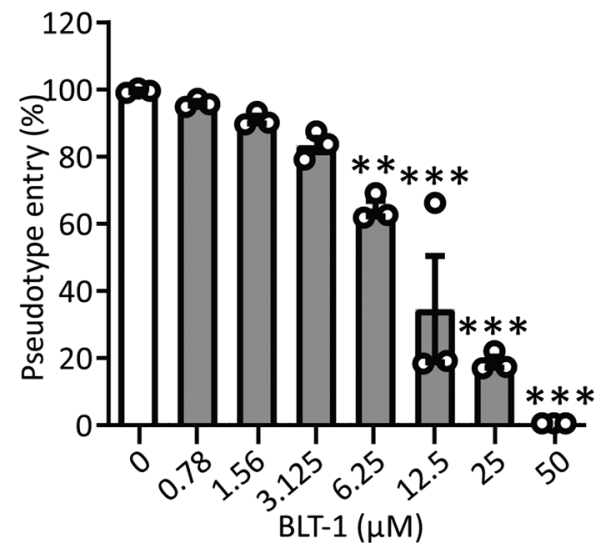


Extended Data Fig. 5 | SR-B1 antagonists block SARS-CoV-2pp infection. a, Immunoblot analysis of SR-B1 expression in Huh-7 cells transfected with HA-SR-B1 or siSR-B1 from Fig. $4 b-d$. b. The bars in the graph represent the RFI from 50 cells per sample from Fig. 4 b. $n=50$ from 3 biologically independent samples and five technical duplication for each sample. $P<0.0001$ for siCtrl vs siSR-B1 by two-tailed Student's t-tests. c, $d$, The percentage of SARS2-S-positive cells (c) and the MFI of SARS-2-S (d) on the cell surface were quantified with isotype and SARS-2-S staining from Fig. 4e. $n=3$ independent biological experiments. P $=0.0475$ for HDL HA-V vs HDL HA-SR-B1 (c), P = 0.0115 for HDL HA-V vs HDL HA-SR-B1 (d) by two-tailed Student's $t$-tests. e, Immunoprecipitation analysis in 293 T cells transfected with Flag-SARS-2-S. The approximate molecular weight $(\mathrm{kDa})$ marker positions are indicated to the left of the blot. $f, g$, Huh-7 cells were preincubated with the indicated concentrations of ITX 5061 (f) or BLT-1 (g) for $48 \mathrm{~h}$, and MTT was added to the cells before OD492 measurements. Signals obtained without compounds were used for normalization. $n=3$ independent biological experiments. Nonsignificant (ns) by one-way ANOVA and Bonferroni's post hoc analysis. h, i, Huh-7 cells preincubated with the indicated concentrations of ITX 5061 (h) or BLT-1 (i) were inoculated with SARS-CoV-2pp in 10\% FBS, and pseudotyped viral entry was analyzed by luciferase activity at $48 \mathrm{~h}$ after infection. Signals obtained without compounds were used for normalization. $n=3$ independent biological experiments. $\mathrm{P}<0.0001$ for $0 \mu \mathrm{M}$ ITX 5061 vs $1.56 \mu \mathrm{M}$ ITX 5061, P < 0.0001 for $0 \mu \mathrm{M}$ ITX 5061 vs $6.25 \mu \mathrm{M}$ ITX 5061, P < 0.0001 for $0 \mu \mathrm{M}$ ITX 5061 vs $25 \mu \mathrm{M}$ ITX $5061, \mathrm{P}<0.0001$ for $0 \mu \mathrm{M}$ ITX 5061 vs $100 \mu \mathrm{M}$ ITX 5061 by one-way ANOVA and Bonferroni's post hoc analysis (h). P $=0.0038$ for $0 \mu \mathrm{M}$ BLT-1 vs $6.25 \mu \mathrm{M} \mathrm{BLT}-1, \mathrm{P}<0.0001$ for $0 \mu \mathrm{M}$ BLT-1 vs $12.5 \mu \mathrm{M}$ BLT-1, $\mathrm{P}<0.0001$ for $0 \mu \mathrm{M}$ BLT-1 vs $25 \mu \mathrm{M}$ BLT-1, P $<0.0001$ for $0 \mu \mathrm{M}$ BLT-1 vs $50 \mu \mathrm{M}$ BLT-1 by one-way ANOVA and Bonferroni's post hoc analysis (i). The data are the mean \pm SEM. ${ }^{\star} P<0.05,{ }^{\star \star} P<0.01,{ }^{\star \star \star} P<0.001$. 


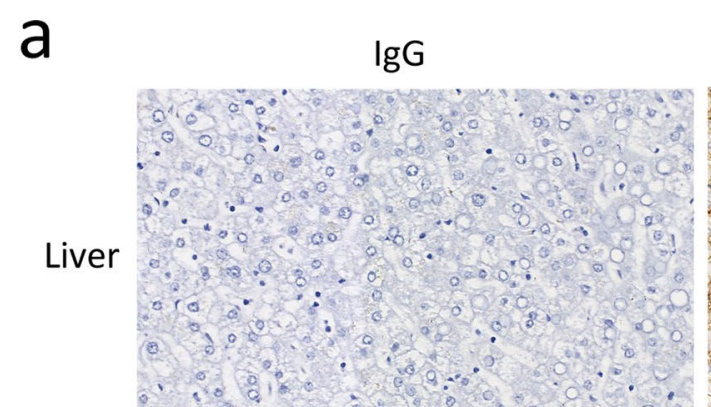

IgG

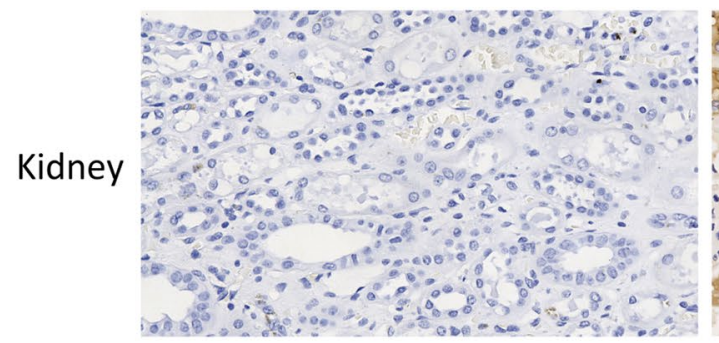

anti-SR-B1

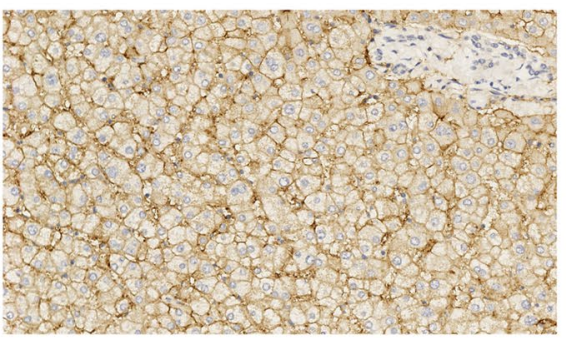

anti-ACE2
anti-SR-B1+His-SR-B1

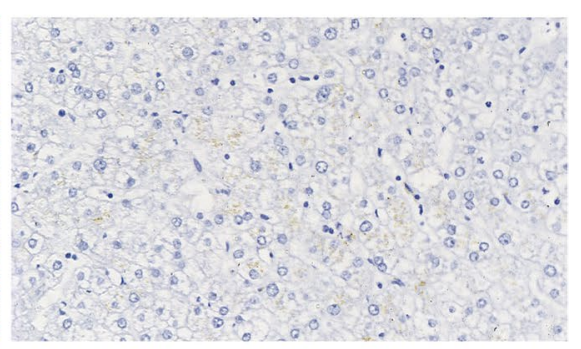

anti-ACE2+His-ACE2 b

Small intestine
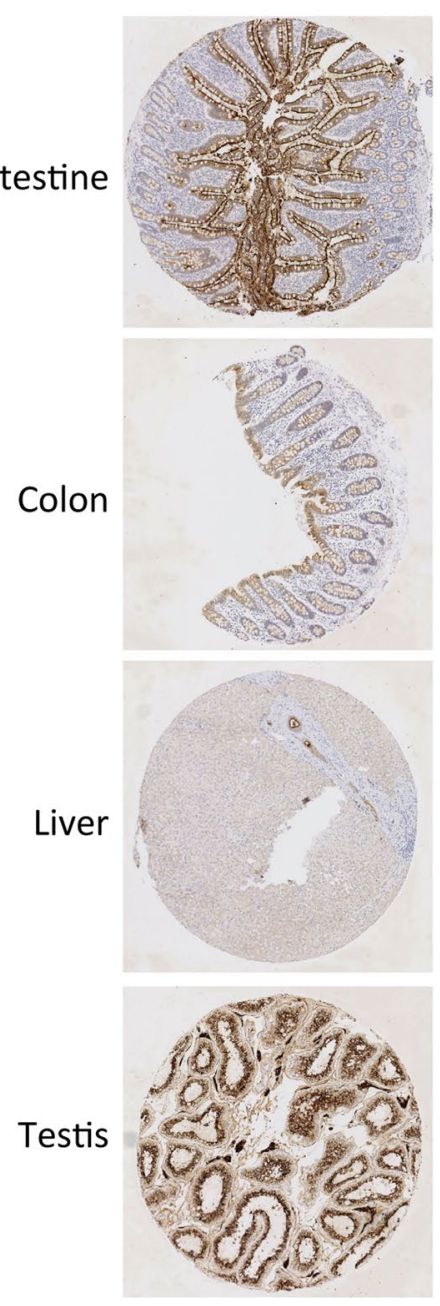

SR-B1
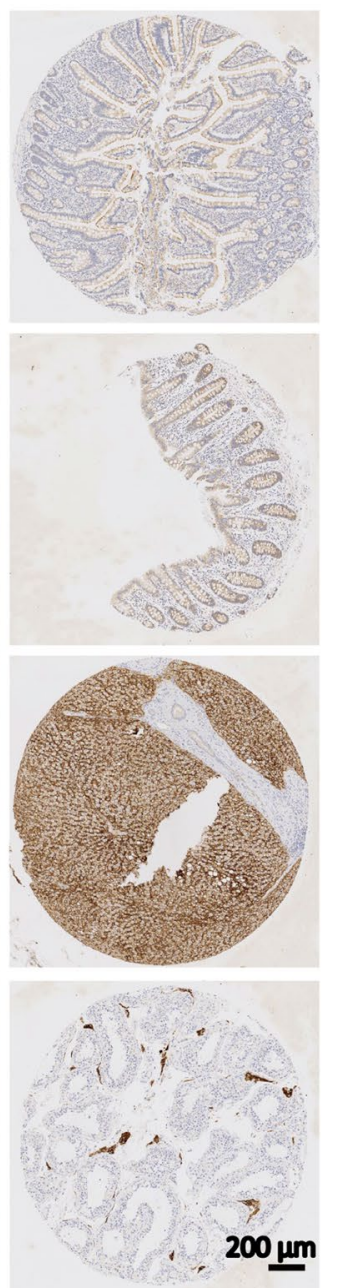
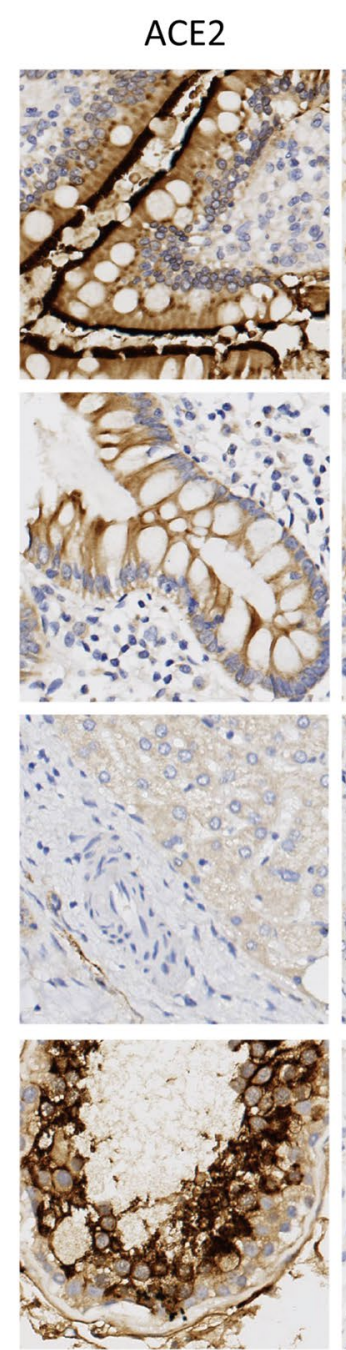

SR-B1
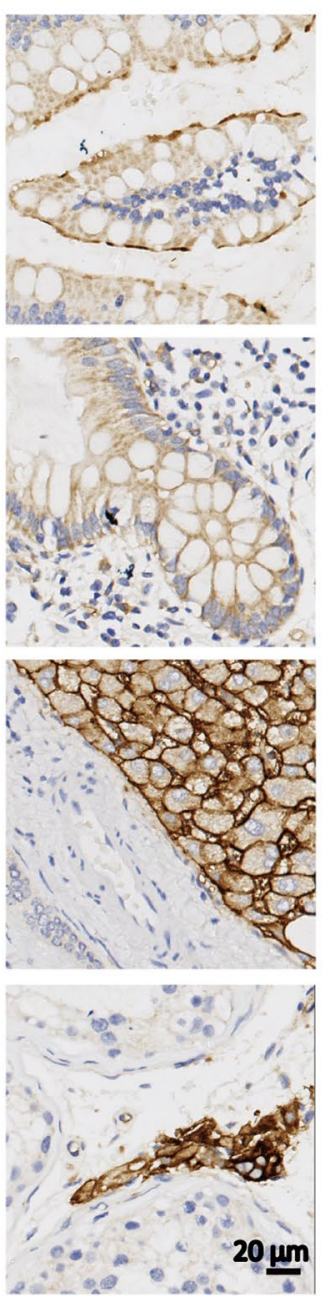

Extended Data Fig. 6 | See next page for caption. 
Extended Data Fig. 6 | Coexpression of SR-B1 and ACE2 in multiple normal human tissues. a, Immunohistochemical staining of liver or kidney tissues incubated with normal IgG, an anti-ACE2 antibody or an anti-SR-B1 antibody. To validate antibody specificity, the anti-ACE2 antibody was preincubated with recombinant His-ACE2 protein and the anti- SR-B1 antibody was preincubated with recombinant His-SR-B1 protein for $1 \mathrm{~h}$ before application to tissue. The results shown are representative of 2 independent biological experiments. $\mathbf{b}$, Representative images of immunohistochemical staining for ACE2 and SR-B1 performed on a paraffin-embedded human normal organ tissue microarray with antibodies against ACE2 or SR-B1; counterstaining with hematoxylin was performed to show nuclei (blue). The scale bars indicate $200 \mu \mathrm{m}$ or $20 \mu \mathrm{m}$, as indicated. The results shown are representative of 2 independent biological experiments. 


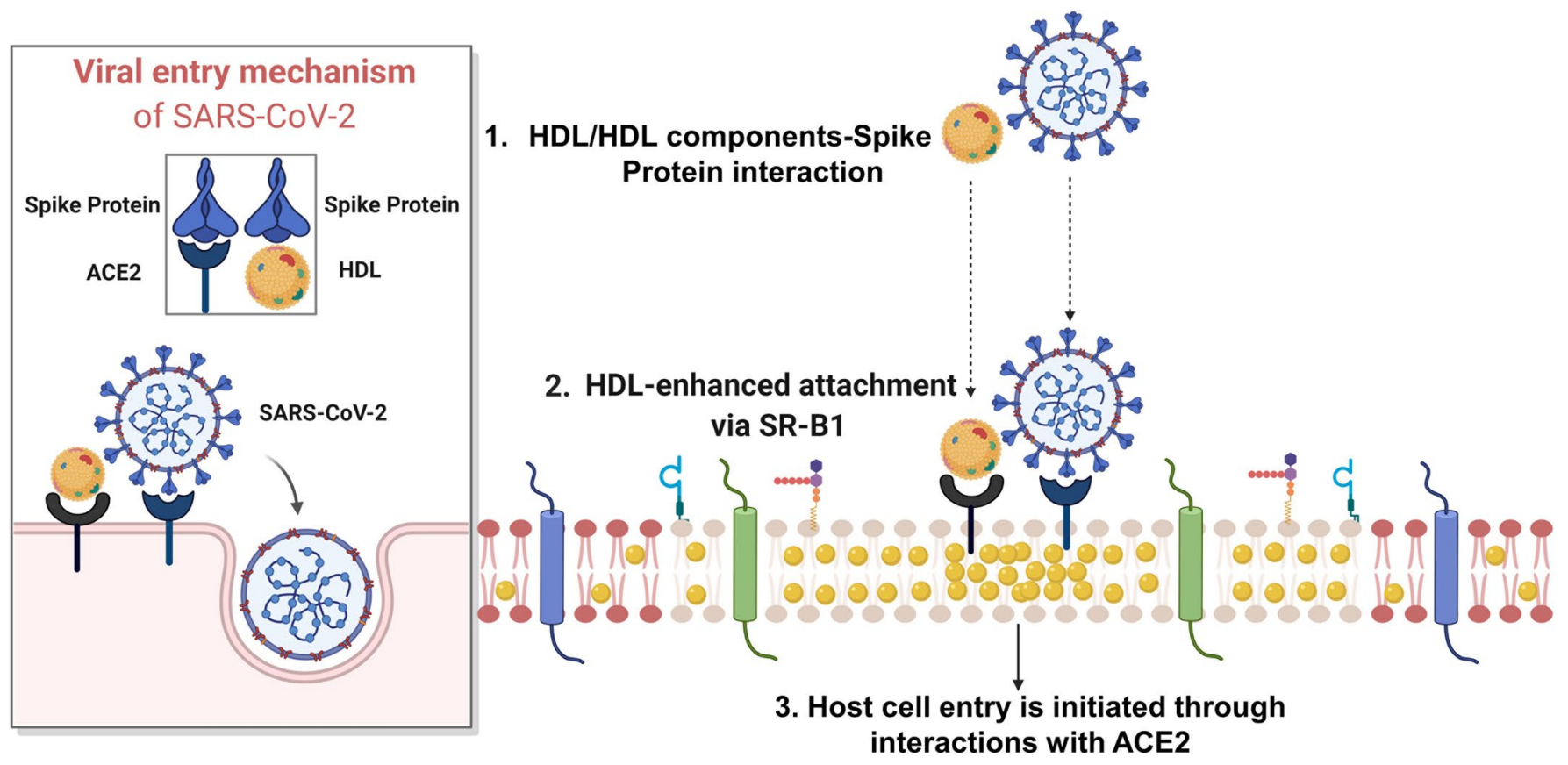

Extended Data Fig. 7 | Hypothetical model of the involvement of SR-B1 in the entry of SARS-CoV-2. Step 1, SARS-2-S binds to cholesterol and HDL or one of its components. Step 2, SR-B1 augments SARS-CoV-2 attachment. Step 3, Host cell entry is initiated through interactions with ACE2. 


\section{natureresearch}

Corresponding author(s): Hui Zhong

Last updated by author(s): Nov 7, 2020

\section{Reporting Summary}

Nature Research wishes to improve the reproducibility of the work that we publish. This form provides structure for consistency and transparency in reporting. For further information on Nature Research policies, see Authors \& Referees and the Editorial Policy Checklist.

\section{Statistics}

For all statistical analyses, confirm that the following items are present in the figure legend, table legend, main text, or Methods section.

n/a Confirmed

$\bigotimes$ The exact sample size $(n)$ for each experimental group/condition, given as a discrete number and unit of measurement

$\square \bigotimes$ A statement on whether measurements were taken from distinct samples or whether the same sample was measured repeatedly

$\square$ The statistical test(s) used AND whether they are one- or two-sided

Only common tests should be described solely by name; describe more complex techniques in the Methods section

Х $\square$ A description of all covariates tested

Х $\square$ A description of any assumptions or corrections, such as tests of normality and adjustment for multiple comparisons

$\triangle$ A full description of the statistical parameters including central tendency (e.g. means) or other basic estimates (e.g. regression coefficient)

AND variation (e.g. standard deviation) or associated estimates of uncertainty (e.g. confidence intervals)

For null hypothesis testing, the test statistic (e.g. $F, t, r$ ) with confidence intervals, effect sizes, degrees of freedom and $P$ value noted

Give $P$ values as exact values whenever suitable.

Х $\square$ For Bayesian analysis, information on the choice of priors and Markov chain Monte Carlo settings

Х $\square$ For hierarchical and complex designs, identification of the appropriate level for tests and full reporting of outcomes

Х $\square$ Estimates of effect sizes (e.g. Cohen's $d$, Pearson's $r$ ), indicating how they were calculated

Our web collection on statistics for biologists contains articles on many of the points above.

\section{Software and code}

Policy information about availability of computer code

Data collection MO Affinity Analysis (version 2.1.23333), Tecan Spark Control (version 2.1), Attune NxT Software (version 3.1.1243.0), Tanon Gelcap (version 5.22), QuantStudio Design \& Analysis Software (version 1.51), Nikon NIS-Elements viewer (version 4.20), OpenSPR Software (version 3.11.6949.28944)

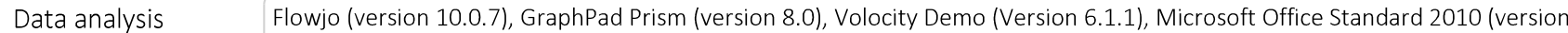
14.0.4760.1000), Nikon NIS-Elements AR (version 4.00.12), TraceDrawer (Version 1.8, Nicoya Lifesciences)

For manuscripts utilizing custom algorithms or software that are central to the research but not yet described in published literature, software must be made available to editors/reviewers. We strongly encourage code deposition in a community repository (e.g. GitHub). See the Nature Research guidelines for submitting code \& software for further information.

\section{Data}

Policy information about availability of data

All manuscripts must include a data availability statement. This statement should provide the following information, where applicable:

- Accession codes, unique identifiers, or web links for publicly available datasets

- A list of figures that have associated raw data

- A description of any restrictions on data availability

The data supporting the findings of this study are available from the corresponding authors upon written request. 


\section{Field-specific reporting}

Please select the one below that is the best fit for your research. If you are not sure, read the appropriate sections before making your selection. $\bigotimes$ Life sciences $\quad \square$ Behavioural \& social sciences $\quad \square$ Ecological, evolutionary \& environmental sciences

For a reference copy of the document with all sections, see nature.com/documents/nr-reporting-summary-flat.pdf

\section{Life sciences study design}

All studies must disclose on these points even when the disclosure is negative.

Sample size No sample size calculations were performed. The sample size ( $n$ ) of each experiment is provided in the corresponding figure captions in the main manuscript and supplementary information files. Sample sizes were chosen to support meaningful conclusions. (PubMed:32494007, 33082294)

Data exclusions No data were excluded

Replication The MST, SPR or peptide-cholesterol binding assays were repeated at least three independent times, all attempts at replication were successful. All infection experiments were repeated at least two independent times, all attempts at replication were successful. The western blot and immunoprecipitation assays were repeated at least two independent times, all attempts at replication were successful. The confocal assays performed at least two independent times, whereas there are at least 3 images for each cell type, all attempts at replication were successful.. The qPCR was performed one time in triplicate all attempts at replication were successful. The flow cytometry assasys were repeated at least two independent times, all attempts at replication were successful.

Randomization Allocation into non-treated or treated conditions was random.

Blinding Technicians were not blinded to group allocation though they were not informed of expected treatment results before and during experimentation. Students blinded their experiments through random numbering of treatment groups and the code was unblinded after analysis.

\section{Reporting for specific materials, systems and methods}

We require information from authors about some types of materials, experimental systems and methods used in many studies. Here, indicate whether each material, system or method listed is relevant to your study. If you are not sure if a list item applies to your research, read the appropriate section before selecting a response.

\begin{tabular}{l|l} 
Materials \& experimental syst \\
\hline $\mathrm{n} / \mathrm{a}$ & Involved in the study \\
\hline & $\bigotimes$ Antibodies \\
$\square$ & Eukaryotic cell lines \\
$\square$ & Palaeontology \\
$\square$ & Animals and other organisms \\
$\square$ Clinical data
\end{tabular}

\begin{tabular}{l|l}
\multicolumn{2}{l}{ Methods } \\
\hline n/a & Involved in the study \\
\hline & $\square$ ChIP-seq \\
$\square$ & $\square$ Flow cytometry \\
$\square$ & $\square$ MRI-based neuroimaging
\end{tabular}

\section{Antibodies}

Antibodies used

a-Tubulin for immunoblotting (Supplier:SigmaAldrich, Cat: T9026, clone: DM1A, Lot: 078M4796 V) Donkey-anti-rabbit antibody conjugated to Alexa Fluor 488 for FACS (Supplier: Biolegend, Cat: 406416, clone: poly4064, Lot: B310664)

SR-B1 antibody for immunoblotting and immunohistochemistry (Supplier: abcam, Cat:ab217318, clone: EPR20190, Lot:GR325004814)

ACE2 antibody for immunoblotting and immunohistochemistry (Supplier: abcam, Cat: ab108252, clone: EPR4435(2), Lot: GR145000-26)

ACE2 antibody for FACS (Supplier: Proteintech, Cat: 21115-1-AP, Lot: 00087165)

PE anti-His antibody for FACS (Supplier: Biolegend, Cat: 362603, clone: J095G46, Lot: B311762)

APC anti-SR-B1 for FACS (Supplier: Biolegend, Cat: 363208, clone: m1B9, Lot: B246109)

Spike antibody for immunoblotting (Supplier: Genetex, Cat: GTX632604, clone:1A9, Lot:42219)

SARS-CoV-2 S1 antibody for confocal microscopy (Supplier: Sino biological, Cat: 40150-T62-COV2, Lot: HD14SE0223) 
cytometry, and referenced in the manuscript

Further validation information of the antibodies by the manufacturer is as follows:

APC anti-SR-B1 for FACS (Supplier: Biolegend, Cat: 363208, clone: m1B9, Lot: B246109) - guaranteed for FACS on website (https://www.biolegend.com/fr-ch/products/apc-anti-human-cd36l1-scarb1-sr-bi-antibody-15058);

PE anti-His antibody for FACS (Supplier: Biolegend, Cat: 362603, clone: J095G46, Lot: B311762) - guaranteed for FACS on website (https://www.biolegend.com/en-us/products/pe-anti-his-tag-antibody-9861);

ACE2 antibody for FACS (Supplier: Proteintech, Cat: 21115-1-AP, Lot: 00087165) - guaranteed for FACS on website (https:// www.ptglab.com/products/ACE2-Antibody-21115-1-AP.htm) and several published applications on website (https:// www.ptglab.com/products/ACE2-Antibody-21115-1-AP.htm);

ACE2 antibody for immunoblotting and immunohistochemistry (Supplier: abcam, Cat: ab108252, clone: EPR4435(2), Lot: GR145000-26) - guaranteed on website (https://www.abcam.com/ace2-antibody-epr44352-ab108252.html) and several published applications on website (https://www.abcam.com/ace2-antibody-epr44352-ab108252.html);

Donkey-anti-rabbit antibody conjugated to Alexa Fluor 488 for FACS (Supplier: Biolegend, Cat: 406416, clone: poly4064, Lot:

B310664) - guaranteed on website (https://www.biolegend.com/en-us/products/alexa-fluor-488-donkey-anti-rabbit-iggminimal-x-reactivity-9380) and several published applications on website (https://www.biolegend.com/en-us/products/alexafluor-488-donkey-anti-rabbit-igg-minimal-x-reactivity-9380);

$\alpha$-Tubulin for immunoblotting (Supplier:SigmaAldrich, Cat: T9026, clone: DM1A, Lot: 078M4796 V) - guaranteed on website (https://www.sigmaaldrich.com/catalog/product/sigma/t9026) and several published applications on website (https:// www.sigmaaldrich.com/catalog/product/sigma/t9026).

\section{Eukaryotic cell lines}

Policy information about cell lines

Cell line source(s)

293T (CRL-3216), Vero E6 (CRL-1568), MDCK (CCL-34) and Hepa 1-6 (CRL-1830) cell lines were from the American Type Culture Collection (ATCC, Rockville, MD, USA). Huh-7 (0403) was from Japanese Collection of Research Bioresources.

Authentication

Cells are purchased from ATCC or Japanese Collection of Research Bioresources.

Mycoplasma contamination

293T, Vero E6, MDCK and Hepa 1-6 cell lines were from the American Type Culture Collection and Huh-7 was from Japanese Collection of Research Bioresources were authenticated by the distributor prior to shipping.

Commonly misidentified lines (See ICLAC register)

No commonly misidentified lines were used.

\section{Human research participants}

Policy information about studies involving human research participants

Population characteristics Immunohistochemical staining for ACE2 and SR-B1 was performed on the paraffin-embedded human normal organ tissue microarray. The human normal organ tissue microarray was purchased from Biomax (MNO661).

Recruitment No human research participants were recruitment.

Ethics oversight

No human research participants were recruitment, and ethics statement was not needed.

Note that full information on the approval of the study protocol must also be provided in the manuscript.

\section{Flow Cytometry}

\section{Plots}

Confirm that:

$\bigotimes$ The axis labels state the marker and fluorochrome used (e.g. CD4-FITC).

Х The axis scales are clearly visible. Include numbers along axes only for bottom left plot of group (a 'group' is an analysis of identical markers).

\All plots are contour plots with outliers or pseudocolor plots.

$\bigotimes$ A numerical value for number of cells or percentage (with statistics) is provided.

\section{Methodology}

Sample preparation

Cells were harvested and resuspended in $100 \mu \mathrm{L}$ phosphatebuffered saline (PBS) containing $0.2 \%$ bovine serum albumin and incubated with the antibodies in dark at room temperature. After 15 minutes, the cells were washed twice with PBS and analyzed on the flow cytometer. For cell surface ACE2 analysis, the cells were incubated with anti-ACE2 antibody for 30 minutes on ice before washing twice followed by incubation with a secondary Alexa Flour 488-conjugated anti-lgG Ab . After 15 minutes, the cells were washed twice with PBS and analyzed on the flow cytometer 
Cell population abundance

Gating strategy cell lines (100\%)

FSC-H vs. SSC-H - gated on cells FSC-H vs. FSC-W - gated on singlets

SSC-H vs. corresponding dyes channel - gated positive on unstained negative control sample.

$\bigotimes$ Tick this box to confirm that a figure exemplifying the gating strategy is provided in the Supplementary Information. 